This item was submitted to Loughborough's Research Repository by the author.

Items in Figshare are protected by copyright, with all rights reserved, unless otherwise indicated.

\title{
Identification and analysis of instability in non-premixed swirling flames using
} LES

PLEASE CITE THE PUBLISHED VERSION

http://dx.doi.org/10.1080/13647830903295899

PUBLISHER

(c) Taylor \& Francis

VERSION

AM (Accepted Manuscript)

LICENCE

CC BY-NC-ND 4.0

REPOSITORY RECORD

Ranga-Dinesh, K.K.J., K.W. Jenkins, M.P. Kirkpatrick, and W. Malalasekera. 2019. "Identification and Analysis of Instability in Non-premixed Swirling Flames Using LES". figshare. https://hdl.handle.net/2134/5494. 
This item was submitted to Loughborough's Institutional Repository (https://dspace.lboro.ac.uk/) by the author and is made available under the following Creative Commons Licence conditions.

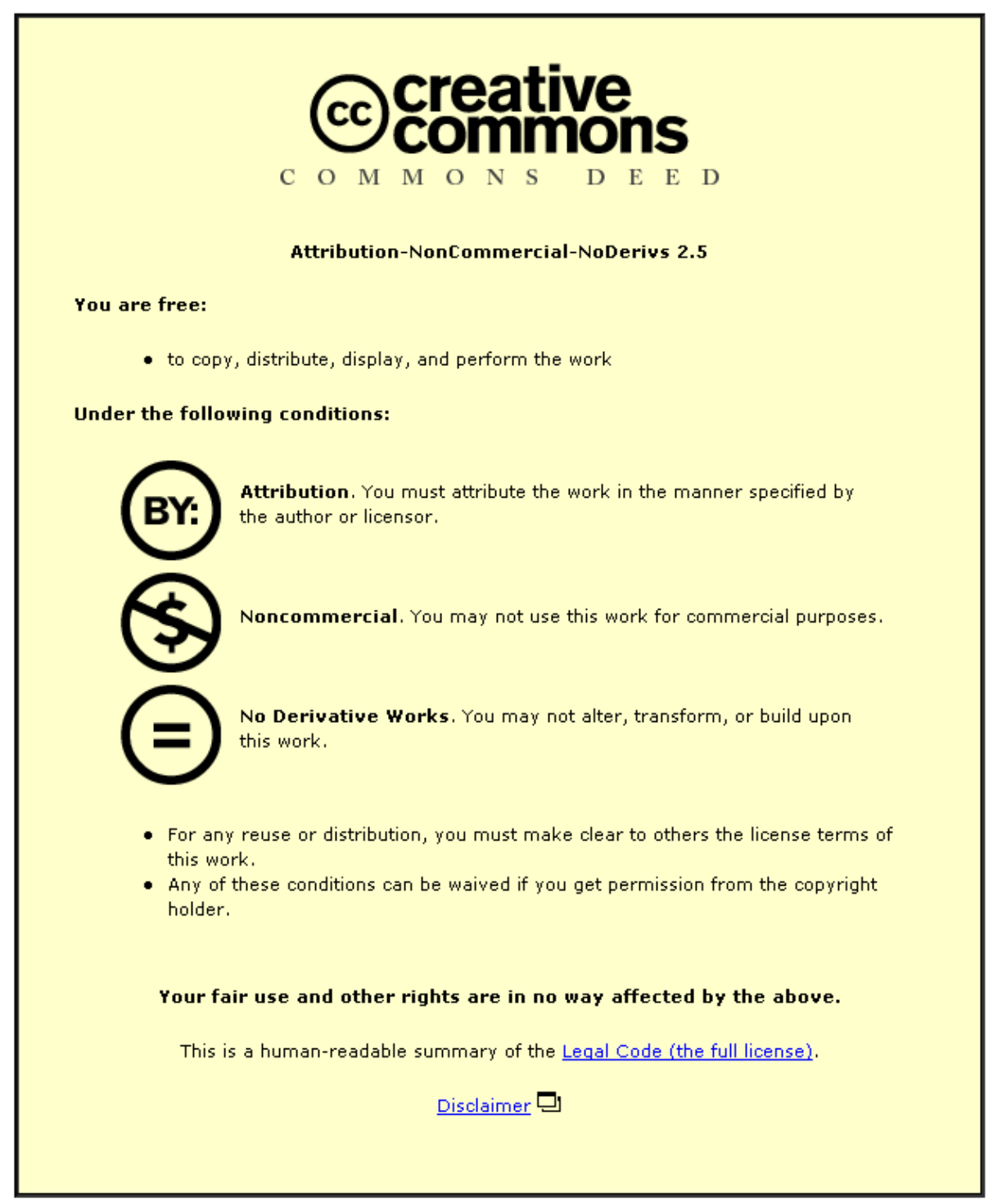

For the full text of this licence, please go to: http://creativecommons.org/licenses/by-nc-nd/2.5/ 


\section{Identification and Analysis of Instability in Non-Premixed Swirling Flames using LES}

K.K.J. Ranga Dinesh ${ }^{1}$, K.W. Jenkins ${ }^{1}$, M.P. Kirkpatrick ${ }^{2}$, W.Malalasekera ${ }^{3}$

1. School Of Engineering, Cranfield University, Cranfield, Bedford, MK43 0AL, UK.

2. School of Aerospace, Mechanical and Mechatronic Engineering, The University of Sydney, NSW 2006, Australia.

3. Wolfson School of Mechanical and Manufacturing Engineering, Loughborough University, Loughborough, Leicester, LE 11 3TU, UK.

Corresponding author: K.K.J.Ranga Dinesh

Email address: Ranga.Dinesh@Cranfield.ac.uk

Postal Address: School of Engineering, Cranfield University, Cranfield, Bedford, MK43 0AL, UK.

Telephone number: +44 (0) 1234750111 ext 5350

Fax number: +44 1234750195

Revised manuscript prepared for the Journal of Combustion Theory and Modelling

$31^{\text {st }}$ of July 2009 


\title{
Identification and Analysis of Instability in Non-Premixed Swirling Flames using LES
}

\author{
K.K.J. Ranga Dinesh ${ }^{1}$, K.W. Jenkins ${ }^{1}$, M.P. Kirkpatrick ${ }^{2}$, W.Malalasekera ${ }^{3}$
}

\begin{abstract}
Large eddy simulations (LES) of turbulent non-premixed swirling flames based on the Sydney swirl burner experiments under different flame characteristics are used to uncover the underlying instability modes responsible for the centre jet precession and large scale recirculation zone. The selected flame series known as SMH flames have a fuel mixture of methane-hydrogen (50:50 by volume). The LES solves the governing equations on a structured Cartesian grid using a finite volume method, with turbulence and combustion modelling based on the localised dynamic Smagorinsky model and the steady laminar flamelet model respectively. The LES results are validated against experimental measurements and overall the LES yields good qualitative and quantitative agreement with the experimental observations. Analysis showed that the LES predicted two types of instability modes near fuel jet region and bluff body stabilized recirculation zone region. The Mode I instability defined as cyclic precession of a centre jet is identified using the time periodicity of the centre jet in flames SMH1 and SMH2 and the Mode II instability defined as cyclic expansion and collapse of the recirculation zone is identified using the time periodicity of the recirculation zone in flame SMH3. Finally frequency spectra obtained from the LES are found to be in good agreement with the experimentally observed precession frequencies.
\end{abstract}

Key words: LES, Swirl, Non-premixed combustion, Precession, Instability modes 


\section{INTRODUCTION}

Swirl based applications in both reacting and non-reacting flows are widely used in many engineering applications to achieve mixing enhancement, flame stabilisation, ignition stability, blowoff characteristics, and pollution reduction. Many engineering applications such as gas turbines, internal combustion engines, burners and furnaces operate in a highly unsteady turbulent environment in which oscillations and instabilities play an important role in determining the overall stability of the system. Although details of oscillations in swirling isothermal and reacting flows have been determined to some extent [1-2], a comprehensive multiscale, multipoint, instantaneous flow structure analysis is still required to access the highly unsteady physical processes that occur in swirl combustion systems. In isothermal swirling flow fields, jet precession, recirculation, VB and a precessing vortex core (PVC) are the main physical flow features that produce instability [3]. However, in combustion systems, these phenomena can promote coupling between combustion, flow dynamics and acoustics [4]. The identification of the oscillation modes and the effect of a PVC on instability remains a challenge especially over a wide range of practical engineering applications. For example, the interactions between different instability oscillations can cause considerable acoustic fluctuations as a result of the pressure field [5-7].

Since the current trend of swirl stabilised combustion systems is shifting towards lean burn combustion to satisfy new emission regulations, combustion instability plays a

vital role and is frequently encountered during the development stage of swirl combustion systems [5]. The most important instability driven mechanisms in gas turbine type combustion configurations can be classified as flame-vortex interactions 
[8-9], fuel/air ratio [10] and spray-flow interactions [11]. Several groups have studied these mechanisms, for example, Richard and Janus [12] and Lee and Santavicca [13] studied the combustion oscillations of a gaseous fuel swirl configuration, $\mathrm{Yu}$ et al. [14] studied the instabilities based on acoustic-vortex flame interactions and Presser et al. [15] studied the aerodynamics characteristics of swirling spray flames for combustion instabilities. Lee and Santavicca [16] and Richards et al. [17] also studied the active and passive control combustion instabilities for gas turbines combustors respectively.

Extensive efforts have gone into performing numerical simulations of swirl stabilised isothermal and reacting systems. Accurate predictions of large scale unsteady flame oscillations, instability modes, PVC structure and the shear layer instability are very demanding and therefore the high-fidelity numerical studies with advanced physical sub-models are necessary. Progress in computing power and physical sub-modelling has led to the expansion of numerical approaches to predict the instabilities in swirl combustion systems [5]. Large eddy simulations (LES) are now widely accepted as a potential numerical tool for solving large scale unsteady behaviour of complex turbulent flows. In LES, the large scale turbulence structures are directly computed and small dissipative structures are modelled. Encouraging results have been reported in recent literature [18-21] which demonstrates the ability of LES to capture the unsteady flow field in complex swirl configurations including multiphase flows and combustion processes such as gas turbine combustion, internal combustion engines, industrial furnaces and liquid-fueled rocket propulsion. 
LES has been successfully used for turbulent non-premixed combustion applications in fairly simple geometries and achieved significant accuracy. For example in gaseous combustion, Cook and Riley [22] applied equilibrium chemistry, and Branley and Jones [23] applied steady flamelet model with single flamelet, Venkatramanan and Pitsch [24] and Kempf et al. [25] used a steady flamelet model with multiple flamelets for LES combustion applications. Pierce and Moin [26] further extended the flamelet model combined with progress variable and developed the so called flamelet/progress variable approach. Navarro-Martinez and Kronenburg [27] have successfully demonstrated the conditional moment closure (CMC) model for LES. Mcmurtry et al. [28] applied the linear eddy model for combustion LES.

Additionally, LES has been used to study swirl stabilised combustion systems in order to investigate the behaviour of flames under highly unsteady conditions. For example, Huang et al. [29] reviewed LES for lean-premixed combustion with a gaseous fuel and analysed details of combustion dynamics associated with swirl injectors. Pierce and Moin [26] performed LES for swirling flames and accurately predicted the turbulent mixing and combustion dynamics for a coaxial combustor. Kim and Syed [30] and Di Mare et al. [31] performed LES calculations of a model gas turbine combustor and found good agreement with experimental measurements. Selle et al. [32] have conducted LES calculations in a complex geometry for an industrial gas turbine burner. Grinstein and Fureby [33] examined the rectangular-shaped combustor corresponding to General Electric aircraft engines using LES and found reasonable agreement with experimental data and Mahesh et al. [34] conducted a series of LES calculations for a section of the Pratt and Whitney gas turbine combustor and validated the LES results against experimental measurements. Fureby et al. [35] 
examined a multi-swirl gas turbine combustor using LES for the design of a future generation of combustors. Bioleau et al. [36] used LES to study the ignition sequence in an annular chamber and demonstrated the variability of ignition for different combustor sectors and Boudier et al. [37] studied the effects of mesh resolution in LES of flow within complex geometries encountered in gas turbine combustors.

The Sydney swirl burner flame series [38-41] effectively allows more opportunities for computational researchers to investigate the complex flow physics and systematic analysis of turbulence chemistry interactions for the laboratory scale swirl burner, which contains features similar to those found in practical combustors. The swirl configuration features a non-premixed flame stabilised by an upstream recirculation zone caused by a bluff body and a second downstream recirculation zone induced by swirl. A few attempts have already been made to model the Sydney swirling flame series using numerous combustion models. Among them El-Asrag and Menon [42] and James et al. [43] modelled flames with different combustion models. In earlier studies, we have shown that LES predicts different isothermal swirling flow fields of the Sydney swirl flame series with a good degree of success [44] and later extended the work to the reacting cases [45]. We have also investigated flame comparisons based on two different independent LES codes [46] and found good agreement especially for capturing the vortex breakdown, recirculation, turbulence and basic swirling flame structures. Despite these contributions and validation studies, a systemic study of flow instabilities associated with the Sydney swirling flames is essential and timely. Ranga Dinesh and Kirkpatrick [47] recently examined the instability of isothermal swirling jets for a wide range of Reynolds and swirl numbers and captured PVC structures, distinct precession frequencies and also found good 
agreement with the experimental observations. Therefore the current work which is a continuation of previous work [47] is focused on capturing the flame oscillations and corresponding instability modes associated with the Sydney swirl burner SMH flame series originally identified by Al-Abdeli et al. [41]. Here, we address the time periodicity in the centre jet and the recirculation zone and the instability modes associated with a centre jet and the bluff body stabilised recirculation zone. This paper is organised as follows: Section 2 describes the mathematical formulations associated with LES and is followed by the simulation details (section 3) and experimental configuration (section 4). In section 5 we discuss the results for all three flames (SMH1, SMH2 and SMH3) from low to high swirl numbers under different flow conditions. Finally, we conclude the work in section 6 and suggest future work.

\section{Mathematical Formulations}

\section{A. Filtered LES equations}

In LES, the most energetic large flow structures are resolved, whereas the less energetic small scale flow structures are modelled. A spatial filter is generally applied to separate the large and small scale structures. For a given function $f(x, t)$ the filtered field $\overline{f(x, t)}$ is determined by convolution with the filter function $G$

$$
\bar{f}(x)=\int_{\Omega} f\left(x^{\prime}\right) G\left(x-x^{\prime}, \bar{\Delta}(x)\right) d x^{\prime},
$$

where the integration is carried out over the entire flow domain $\Omega$ and $\bar{\Delta}$ is the filter width, which varies with position. A number of filters are used in LES such as top hat or box filter, Gaussian filter, spectral filter. In the present work, a so called top hat filter (implicit filtering) having a filter-width $\bar{\Delta}_{j}$ proportional to the size of the local cell is used. In turbulent reacting flows large density variations occur, which are 
treated using Favre filtered variables, which leads to the transport equations for Favre filtered mass, momentum and mixture fraction:

$$
\begin{gathered}
\frac{\partial \bar{\rho}}{\partial t}+\frac{\partial \bar{\rho} \tilde{u}_{j}}{\partial x_{j}}=0 \\
\frac{\partial \bar{\rho} \tilde{u}_{i}}{\partial t}+\frac{\partial\left(\bar{\rho} \tilde{u}_{i} \tilde{u}_{j}\right)}{\partial x_{j}}=-\frac{\partial \bar{P}}{\partial x_{i}}+\frac{\partial}{\partial x_{j}}\left[\overline{2 \rho}\left(v+v_{t}\right)\left[\frac{1}{2}\left(\frac{\partial \tilde{u}_{i}}{\partial x_{j}}+\frac{\partial \tilde{u}_{j}}{\partial x_{i}}\right)-\frac{1}{3} \delta_{i j} \frac{\partial \tilde{u}_{k}}{\partial x_{k}}\right]\right] \\
+\frac{1}{3} \frac{\partial}{\partial x_{j}}\left[\bar{\rho} \delta_{i j} \tau_{k k}\right]+\bar{\rho} g_{i} \\
\frac{\partial \bar{\rho} \tilde{f}}{\partial t}+\frac{\partial}{\partial x_{j}}\left(\bar{\rho} \tilde{u}_{j} \tilde{f}\right)=\frac{\partial}{\partial x_{j}}\left[\bar{\rho}\left(\frac{\tilde{v}}{\sigma}+\frac{v_{t}}{\sigma_{t}}\right) \frac{\partial \tilde{f}}{\partial x_{j}}\right]
\end{gathered}
$$

In the above equations $\rho$ represents the density, $u_{i}$ is the velocity component in $x_{i}$ direction, $p$ is the pressure, $v$ is the kinematics viscosity, $f$ is the mixture fraction, $v_{t}$ is the turbulent viscosity, $\sigma$ is the laminar Schmidt number, $\sigma_{t}$ is the turbulent Schmidt number and $\tau_{k k}$ is the isotropic part of the sub-grid scale stress tensor. An over-bar describes the application of the spatial filter while the tilde denotes Favre filtered quantities. The laminar Schmidt number was set to 0.7 and the turbulent Schmidt number for mixture fraction was set to 0.4 . Finally to close these equations, the turbulent eddy viscosity $v_{t}$ in Eq. (3) and (4) has to be evaluated using a model equation.

\section{B. Modelling of turbulent eddy viscosity}

The Smagorinsky eddy viscosity model [48] is employed to calculate the turbulent eddy viscosity $v_{t}$. The Smagorinsky eddy viscosity model [48] uses a model parameter $C_{s}$, the filter width $\bar{\Delta}$ and strain rate tensor $S_{i, j}$ such that 


$$
v_{t}=C_{s} \bar{\Delta}^{2}\left|S_{i, j}\right|=C_{s} \bar{\Delta}^{2}\left|\frac{1}{2}\left(\frac{\partial \tilde{u}_{i}}{\partial x_{j}}+\frac{\partial \tilde{u}_{j}}{\partial x_{i}}\right)\right|
$$

The model parameter $C_{s}$ is obtained using the localised dynamic procedure of Piomelli and Liu [49].

\section{Modelling of combustion}

In LES, chemical reactions occur at the sub-grid scales and therefore modelling is required for combustion chemistry. Here an assumed probability density function (PDF) for the mixture fraction is chosen as a means of modelling the sub-grid scale mixing with $\beta$ PDF used for the mixture fraction. The functional dependence of the thermo-chemical variables is closed through the steady laminar flamelet approach. In this approach the variables such as density, temperature and species concentrations depend on Favre filtered mixture fraction, mixture fraction variance and scalar dissipation rate. The sub-grid scale variance of the mixture fraction is modelled using the gradient transport model. The flamelet calculations were performed using the Flamemaster code developed by Pitsch [50], which incorporates the GRI 2.11 mechanism with detailed chemistry [51].

\section{Simulation Details}

In the current work all simulations are performed using the PUFFIN code developed by Kirkpatrick et al. [52-54] and later extended by Ranga Dinesh [55]. PUFFIN computes the temporal development of large-scale flow structures by solving the transport equations for the Favre-filtered continuity, momentum and mixture fraction. The equations are discretised in space with the finite volume formulation using Cartesian coordinates on a non-uniform staggered grid. Second order central 
differences (CDS) are used for the spatial discretisation of all terms in both the momentum equation and the pressure correction equation. This minimizes the projection error and ensures convergence in conjunction with an iterative solver. The diffusion terms of the scalar transport equation are also discretised using the second order CDS. However, discretisation of convection term in the mixture fraction transport equation using CDS would cause numerical wiggles in the mixture fraction. To avoid this problem, here we employed a Simple High Accuracy Resolution Program (SHARP) developed by Leonard [56].

In order to advance a variable density calculation, an iterative time advancement scheme is used. First, the time derivative of the mixture fraction is approximated using the Crank-Nicolson scheme. The flamelet library yields the density and calculates the filtered density field at the end of the time step. The new density at this time step is then used to advance the momentum equations. The momentum equations are integrated in time using a second order hybrid scheme. Advection terms are calculated explicitly using second order Adams-Bashforth while diffusion terms are calculated implicitly using second order Adams-Moulton to yield an approximate solution for the velocity field. Finally, mass conservation is enforced through a pressure correction step. Typically 8-10 outer iterations of this procedure are required to obtain satisfactory convergence at each time step. The time step is varied to ensure that the Courant number $C_{o}=\Delta t u_{i} / \Delta x_{i}$ remains approximately constant where $\Delta x_{i}$ is the cell width, $\Delta t$ is the time step and $u_{i}$ is the velocity component in the $x_{i}$ direction. The solution is advanced with a time step corresponding to a Courant number in the range of $C_{o}=0.3$ to 0.6. The Bi-Conjugate Gradient Stabilized 
(BiCGStab) method with a Modified Strongly Implicit (MSI) preconditioner is used to solve the system of algebraic equations resulting from the discretisation.

Simulations for the flames SMH1 and SMH2 were carried out with the dimensions of $300 \times 300 \times 250 \mathrm{~mm}$ in the $\mathrm{x}, \mathrm{y}$ and $\mathrm{z}$ directions respectively and employed nonuniform Cartesian grids with 3.4 million cells. Since the flame SMH3 has high fuel jet velocity, it produces a longer flame than both the SMH1 and SMH2 in the streamwise direction, we therefore used a larger domain for the axial direction such that $300 \times 300 \times 400 \mathrm{~mm}$ which employed 4 million cells.

The mean axial velocity distribution for the fuel inlet and mean axial and swirling velocity distributions for air annulus are specified using power low profiles;

$$
<U>=1.218 U\left(1-\frac{|y|}{\delta}\right)^{1 / 7}
$$

where $U_{j}$ is the bulk velocity, $y$ is the radial distance from the jet centre line and $\delta=1.01 R_{j}$, where $R_{j}$ is the fuel jet radius of $1.8 \mathrm{~mm}$. The factor 1.01 is included to ensure that velocity gradients are finite at the walls. The same equation is used for the swirling air stream with $U_{j}$ replaced by bulk axial velocity $U_{s}$ and bulk tangential velocity $W_{s}$ and $y$ being the radial distance from the centre of the annulus and $\delta=1.01$ times the half width of the annulus.

Velocity fluctuations are generated from a Gaussian random number generator, which are then added to the mean velocity profiles such that the inflow has the same turbulence kinetic energy levels as that obtained from the experimental data. A top hat profile is used as the inflow condition for the mixture fraction. A Free slip boundary 
condition is applied at the solid walls and at the outflow plane, a convective outlet boundary condition is used for the velocities and a zero normal gradient condition is used for the mixture fraction. All computations were carried out for a sufficient time to ensure we achieved converged solutions, and the total time for each simulation is $0.24 \mathrm{~s}$.

\section{Experimental Configuration}

The Sydney swirl burner configuration shown in Figure 1, which is an extension of the well-characterized Sydney bluff body to the swirling flames. Extensive details have been reported in the literature for the Sydney swirling flames including flow field and compositional structures for pure methane flames [38], stability characteristics [39], compositional structure [40] and time varying behaviour [41].

The burner has a 60mm diameter annulus for a primary swirling air stream surrounding a circular bluff body of diameter $\mathrm{D}=50 \mathrm{~mm}$ and the central fuel jet is 3.6mm in diameter. The burner is housed in a secondary co-flow wind tunnel with a square cross section with $130 \mathrm{~mm}$ sides. Swirl is introduced aerodynamically into the primary annulus air stream at a distance 300mm upstream of the burner exit plane and inclined 15 degrees upward to the horizontal plane. The swirl number can be varied by changing the relative magnitude of the tangential and axial flow rates. The literature already includes the details of flame conditions and can be found in [38-41].

In the present LES calculations, the SMH flames were modelled burning a methanehydrogen fuel mixture (50:50 by volume). The properties of the simulated flames are

summarised in table 1 . Here, $U_{j}(\mathrm{~m} / \mathrm{s}), U_{s}(\mathrm{~m} / \mathrm{s}), W_{s}(\mathrm{~m} / \mathrm{s}), U_{e}(\mathrm{~m} / \mathrm{s}), S_{g}$, and 
Re are fuel jet velocity, axial velocity of the primary annulus, swirl velocity of the primary annulus, secondary co-flow velocity, swirl number and Reynolds number of the fuel jet respectively.

\begin{tabular}{|l|l|l|l|l|l|l|l|}
\hline Case & Fuel & $U_{j}(\mathrm{~m} / \mathrm{s})$ & $U_{s}(\mathrm{~m} / \mathrm{s})$ & $W_{s}(\mathrm{~m} / \mathrm{s})$ & $U_{e}(\mathrm{~m} / \mathrm{s})$ & $S_{g}$ & $\mathrm{Re}$ \\
\hline SMH1 & $\mathrm{CH}_{4}-\mathrm{H}_{2}$ & 140.8 & 42.8 & 13.8 & 20.0 & 0.32 & 19,300 \\
\hline SMH2 & $\mathrm{CH}_{4}-\mathrm{H}_{2}$ & 140.8 & 29.7 & 16.0 & 20.0 & 0.54 & 19,300 \\
\hline SMH3 & $\mathrm{CH}_{4}-\mathrm{H}_{2}$ & 226.0 & 29.7 & 16.0 & 20.0 & 0.54 & 31,000 \\
\hline
\end{tabular}

Table 1. Details about the characteristics properties of SMH flame series

\section{Results and Discussion}

The Sydney swirl burner is designed to study the reacting and non-reacting swirling flow structures for a range of swirl, Reynolds numbers and fuel mixtures. The aim here is to uncover the time periodicity in the centre jet and bluff body stabilised recirculation zone and the corresponding precession frequencies while identifying the dominant instability modes for all three swirling flames. Here we have considered the SMH flame series which contains three different swirling flames known as SMH1, SMH2 and SMH3 for three different Reynolds and swirl numbers [39-41]. Since validation of LES results with experimental measurements is necessary, first we address the comparisons between LES computations and the experimental measurements. The second part is a discussion of the existence of instability modes in which we analyse the time periodicity in both the centre jet and bluff body stabilised recirculation zone. 


\subsection{Validation studies}

Figures 2-4 show snapshots of filtered temperatures for SMH1, SMH2 and SMH3 respectively. The high temperature distribution region in the bluff body stabilized recirculation zone is much wider for both SMH1 and SMH3 flames thinner for the SMH2 flame. The strong and weak neck zones are visible in SMH1 and SMH3 respectively. The neck zones of flames SMH1 and SMH3 appear approximately 60mm and 50mm downstream from the burner exit plane respectively.

Shown in Figure 5 is the time averaged mean axial velocity at different axial locations. The comparisons are presented for $\mathrm{x} / \mathrm{D}=0.2,0.8,1,6$ and 3.5 for SMH1 (left side) and $\mathrm{x} / \mathrm{D}=0.136,0.4,1.2$ and 2.5 for $\mathrm{SMH} 2$ (right side). The experimental data shows that for both flames the bluff body stabilised recirculation zone is extending axially up to $\mathrm{x} / \mathrm{D}=1.2$ from the burner exit plane [39]. The occurrence of the negative mean axial velocity values at $\mathrm{x} / \mathrm{D}=0.136,0.2,0.4$ and 0.8 indicates a flow reversal which is well captured by the LES. However, the calculations over estimate the centerline mean axial velocity at $\mathrm{x} / \mathrm{D}=1.6,3.5$ for $\mathrm{SMH} 1$ and at $\mathrm{x} / \mathrm{D}=2.5$ for $\mathrm{SMH}$. This is most likely attributed to the difference in momentum decay in the central jet with the LES centre jet breakdown slower than that found in the experiment.

Figure 6 shows comparison between numerical and experimental results for the time averaged mean swirling velocity (left side: SMH1, right-side: SMH2). The comparison between calculations and measurements are very good for flame SMH1 and reasonably good for flame SMH2. The LES results captured well the peak values which appear in the shear layers between the centre jet and the recirculation zone and also the outer flow region and the recirculation zone. However, the mean swirl 
velocity of SMH2 (right side) has some over prediction at $\mathrm{x} / \mathrm{D}=1.2$ and 2.5 and this may be attributed to the differences of swirl momentum decay in experimental and numerical results. Comparison between LES calculations and the experimental measurements for the rms (root mean square) axial velocity is shown in Figure 7. The LES results under predict at $\mathrm{x} / \mathrm{D}=0.2,0.8$ and over predict at $\mathrm{x} / \mathrm{D}=1.6,3.5$ for flames SMH1 and SMH2 respectively.

Finally, Figures 8-10 show the comparison of the mean temperature, $\mathrm{CO}_{2}$ and $\mathrm{CO}$ mass fractions (left side: SMH1, right-side: SMH2). Despite the complexity of the flow field, the comparison of the temperature field is reasonable at most axial locations for both flames. The $\mathrm{CO}_{2}$ (Figure 9) profiles follow the same behaviour as temperature for both flames where the LES over predicts the $\mathrm{CO}_{2}$ value at $\mathrm{x} / \mathrm{D}=0.2$. Similar to the temperature distributions, the computed $\mathrm{CO}_{2}$ under predicts at $\mathrm{x} / \mathrm{D}=0.8$ and the peak values are not accurately captured for the SMH1 flame. For CO (Figure 10), the radial spread is underestimated only at $x / D=0.2$ for both flames. Further downstream the comparisons are very good. Given the complexity of the flame and flow field, LES calculated species concentrations are in good agreement with the experimental data.

In summary, the flames studied here involve a complex flow situation and flame structures in which there occurs recirculation, centre jet precession, shear layer instability and complex turbulence chemistry interactions. The computed flow and scalar patterns agree well with the experimental data and hence this validation allows us to succeed our main goal "identification of instability modes associated with SMH flame series”. 


\subsection{Time periodicity in the centre jet of flame SMH1}

This section discusses the centre jet precession of flame SMH1 using the LES data that has been originally identified by Al-Abdeli et al. [41] in their experimental investigation for the Sydney swirl burner experimental data base managed by Masri's group [40].

Figure 11 (a-h) shows a series of snapshots (filtered axial velocity) at different periodic time intervals generated from the LES. From the experimental work, AlAbdeli et al. [41] observed a periodic (cyclic) precession motion of the centre jet for flame SMH1 which was defined as Mode I instability using snapshots at different time periods. Similarly here we used the snapshots of filtered axial velocity to demonstrate the Mode I instability in flame SMH1.

The calculated images from (a) to (h) in Figure 11 show the periodic variation of the centre jet. For example, Figure 11 (a) shows a snapshot of the filtered axial velocity which is vertical at that particular time. Then slowly starts to shift towards one side of the centerline (b-c) and then return again in (d). The centre jet is then seen to cross over to other side (e-g), and finally reaching the starting position of (a) in the last snapshots (h). Since Mode I instability is believed to be a consequence of an orbital (circular) motion about the central axis the observations from Figure 11 can be defined as Mode I instability. It is important to note that the Mode I instability has also been identified in Sydney isothermal swirling flows both numerically by Ranga Dinesh and Kirkpatrick [47] and experimentally by Al-Abdeli and Masri [57]. In the current case, vortex shedding due to hydrodynamic instability might be the same as that is in the isothermal case, but the complexity of the Mode I instability increases 
due to combustion heat release, which eventually increases the unsteady frequencies. The visualisation of Mode I instability of flame SMH1 in a plane perpendicular to the centreline is shown in Figure 12. Figures 12 aa, cc and gg show the snapshots of the filtered axial velocity in a normal plane correspond to Figures $11 \mathrm{a}$, c and g. The axial location of the considered horizontal plane for Figure 12 is marked by a line in Figure 11 c. Figures 11 and 12 indicate that the swirling motion rotates the centre jet around the axis as cyclic motion and thus forms PVC structures in the near region of the jet. A further investigation to determine the changes of the heat release respect to centre jet precession frequency for different swirl numbers should be performed to investigate the rapid changes in the instantaneous temperature field.

In order to analyse the time periodicity in the centre jet, a spatial jet locator must be considered and here we have a spatial jet locator which is positioned just off the burner centerline such that $\mathrm{x}=12.3 \mathrm{~mm}$ (axial location) and $\mathrm{r}=2.3 \mathrm{~mm}$ (radial location) which is similar to the experimental location [41]. A pair of monitoring points either side of the centre jet are considered, and we constructed a power spectrum by applying the Fast Fourier Transform (FFT) for the instantaneous filtered axial velocity. Figure 13 shows the power spectrum of SMH1 at the spatial jet locator with several peaks at low frequency levels and peaks become more discrete and appear around $\sim 50 \mathrm{~Hz}$. Al-Abdeli et al. [41] used three different experimental techniques to detect distinct frequencies such as Laser Mie scattering, Shadowgraphs and LDV (Laser Doppler Velocimetry) spectra. For SMH1 flame, Al-Abdeli et al. [41], detected distinct frequencies for Mode I instability such that $\sim 47 \mathrm{~Hz}$ from Laser Mie scattering, $\sim 47 \mathrm{~Hz}$ from Shadowgraphs and $41-58 \mathrm{~Hz}$ from LDV spectra (Laser 
Doppler Velocimetry). Therefore the distinct frequency found by LES calculation $(\sim 50 \mathrm{~Hz})$ is in agreement with that found in the experimental investigation.

\subsection{Time periodicity in the centre jet of flame SMH2}

Snapshots of the filtered axial velocity for eight different time periods from the LES are shown in Figure 14 (a-h). The SMH2 flame has relatively higher swirl number ( $S_{g}=0.54$ ) than SMH1 and hence it exists a higher centrifugal force than that in flame SMH1. Again, Al-Abdeli et al. [41] found a cyclic variation of the centre jet which defined as Mode I instability. Figure 14 (a) indicates a vertical (straight) centre jet which then starts to move to one side till Figure 14 (c). The jet then starts to move to other side from Figure 14 (e) and finally move back to its initial position in Figure 14 (h). Since LES has captured this periodic centre jet precession we can refer this motion as Mode I instability [41]. Furthermore, as seen in Figure 14 the centre jet of flame SMH2 appears to shift more radially in both directions than that found in SMH1. This can be expected since SMH2 has high centrifugal force than SMH1 due to the high swirl number. Figure 15 shows the occurrence of Mode I instability of flame SMH2 in a plane perpendicular to the centreline. Figures 15 aa, cc and ff show the cross sectional snapshots corresponding to Figures 14 a, c and f respectively. The location of the horizontal plane has been marked by a line in Figure 14 c. As seen in Figure 15 snapshots of the filtered axial velocity in a horizontal plane (normal to snapshots in Figure 11) again exhibit precession behaviour.

The power spectrum of flame SMH2 at the spatial jet locator defined in the previous section is shown in Figure 16. Similar to SMH1, the power spectrum of flame SMH2 also shows peaks at low frequency levels with the peaks becoming more distinct 
around $\sim 47 \mathrm{~Hz}$. The distinct precession frequencies from the simulation demonstrate the Mode I instability of flames SMH2 given by the cyclic variation of the centre jet. Again, Al-Abdeli et al. [41] experimentally found distinct precession frequencies for Mode I instability such that $\sim 55 \mathrm{~Hz}$ from Laser Mie scattering, $\sim 55 \mathrm{~Hz}$ from Shadowgraphs and $48-58 \mathrm{~Hz}$ from LDV spectra.

\subsection{Time periodicity in the recirculation zone of flame SMH3}

Here we discuss the time varying behaviour of flame SMH3 as well as another Mode of instability this time associated with bluff body stabilised recirculation zone originally identified by Al-Abdeli et al. [41]. It is important to note that the Mode II instability was only identified for a few of the Sydney swirling flames depend on the conditions that have been adopted to produce the flame. The Mode II instability appears to be rather weak, but can still be seen for SMH3 flame. The cyclic oscillation which appears in flame SMH3 defined as Mode II which can be described as the expansion and collapse of the bluff body stabilised recirculation zone which is also known as “puffing” motion.

To demonstrate the Mode II instability based on the LES data, we use six snapshots of the filtered axial velocity (Figure 17 (a-f)) which only shows the negative axial velocity which clearly indicates the region of the upstream recirculation zone at different time periods. In addition, we generated the power spectrum for a particular location at the envelope of the recirculation zone. Figure 17 shows the contour plots of filtered axial velocity for the bluff body stabilised recirculation zone, where a solid line indicates the boundary of the recirculation zone and the dashed lines indicate the negative filtered axial velocity inside the recirculation zone. The ranges of contour 
values $(0 \mathrm{~m} / \mathrm{s}$ to $-20 \mathrm{~m} / \mathrm{s})$ are shown in Figure 17 (a). Figure 17 (a) at one particular time shows a recirculation zone which continues to reduce over the next two time intervals shown in Figures 17 (b) and (c) respectively. The recirculation zone then starts to expand in Figure 17 (d) and (e) and eventually forms a similar shape as initial snapshot in Figure 17 (f). Thus the time dependent snapshots show a sequential collapse/contraction and then expansion of the bluff body stabilised recirculation zone similar to that found in the experimental observation and can be referred as "puffing" motion, defined as Mode II instability [41]. The expansion and collapse of the bluff body stabilised recirculation zone has only been identified in some of the Sydney swirling flames including SMH3. This might occur due to coupling between combustion heat release and flow velocities that have been adopted. Furthermore, the Mode II instability has not been identified in Sydney swirling isothermal flow fields either numerically [47] or experimentally [57]. Hence the identification of Mode II instability (extension and collapse of the bluff body stabilised recirculation zone) both numerically and experimentally can add a new dimension to the already existing physical aspects of swirling flows [3] and thus help to derive a correlation between flow reversal, mixing rate and temperature gradient. However, more investigation is still needed both numerically and experimentally to reveal a major correlation for this finding and we are keen to extend the present work to derive a mechanism for the combustion instability based on flow reversal, mixing rate and temperature gradient specifically for the flame SMH3.

Figure 18 shows the power spectrum of SMH3 at the envelope of the bluff body stabilised recirculation zone. In order to analyse the time periodicity in this case the recirculation zone, a pair of monitoring points around the envelope of the bluff body 
stabilised recirculation zone are considered, which is similar to the experimental case [41]. Again, the power spectrum is constructed by applying the Fast Fourier Transform (FFT) for the instantaneous filtered axial velocity. The power spectrum of flame SMH3 shows some peaks at low frequency levels and peaks become more distinct around $\sim 40-60 \mathrm{~Hz}$. The peaks around $\sim 40-60 \mathrm{~Hz}$ are attributed to the Mode II instability, and the identification of these peaks of Mode II unsteadiness further demonstrates the usefulness of the LES technique for simulation of complex unsteady flames and combustion dynamics. The experimental group revealed [41] the distinct precession frequencies for Mode II instability such that shadowgraphs found the distinct precession frequency of $\sim 60 \mathrm{~Hz}$ and LDV spectra found the range $63-67 \mathrm{~Hz}$.

\section{Conclusions}

We have performed LES of turbulent swirl flames known as SMH1, SMH2 and SMH3 and investigated the instabilities associated with each flames experimentally conducted by Masri and co-workers [39-41]. The LES technique was applied successfully to study the flame oscillations and instability modes originally identified by Al-Abdeli et. al. [41].

First we compared the LES results with experimental data and found that results are in good agreement for both velocity and scalar fields. Then we investigated the instability mechanisms associated with the SMH flame series. The unsteady data from the simulations along with the Fast Fourier Transform (FFT) algorithm have been used for data analysis. Various snapshots and power spectra indicate the time periodicity in the centre jet and the time periodicity in the recirculation zone. The simulations captured the Mode I instability for both SMH1 and SMH2 flames in 
which the instability involves precession of the centre jet. The power spectra produced at the spatial jet locator further demonstrates the link between precession frequencies and the Mode I instability for both flames. Another Mode of instability is shown to be associated with large scale unsteadiness of the recirculation zone characterized by “puffing” motion in flame SMH3. This has been referred as Mode II instability and has not been identified in isothermal jets [47][57]. The identification of two types of instability modes associated with these flames demonstrates that the LES technique is useful and promising tool to capture the instabilities in complex turbulent non-premixed flames. Although we have performed all simulations in incompressible pressure based low Mach number variable density algorithm, the effect of compressibility cannot be ruled out as a results of high fuel jet velocities for all three flames. Therefore, the numerically computed pressure oscillations and the heat release patterns might have some discrepancies with experimental values and thus make differences for the distinct precession frequencies as appeared in the power spectra. In addition, the rate of energy transfer to a fluctuation depends on the fluctuation itself and this may be able to deviate the computed distinct frequencies with experimentally observed values. However, the identification of Mode I and II instabilities both computationally and experimentally provide useful details for the presence of instabilities in turbulent non-premixed swirling flames and also highlights the differences compared with the hydrodynamic instability.

The future numerical work will study the effect of swirl on combustion dynamics which will lead to the identification of more flow features and coupling relations between vortex breakdown, turbulence intensity, flame temperature and more 
importantly the behaviour of the already existing instability modes bearing in mind that the excessive swirl may lead to the occurrence of flame flashback.

Since the combustion phenomena in LES acts in small scales the energy transfer from large scale to small scale along with heat release can be critically affected by the swirl number. In such situations, the flame is anchored by the recirculating flow and occurrence of the precession motion as observed in this investigation might cause intermittency in the temperature field. An effort is currently underway to fully investigate the effect of swirl on combustion intermittency for this burner configuration and results will be reported in the near future. 


\section{References}

[1] R.C. Chanaud, Observations of oscillatory motion in certain swirling flows, J. Fluid. Mech. (1965) 21(1), pp.111-121

[2] N. Syred, J.M. Beer, Combustion in swirling flows: a review, Combust. Flame (1974) 23, pp.143-201

[3] N. Syred, A review of oscillation mechanisms and the role of the precessing vortex core (PVC) in swirl combustion systems. Prog. Energy. Combust. Sci. (2006) ,32, pp. 93-161

[4] D. Froud, T. O’Doherty, N. Syred, Phase averaging of the precessing vortex core in a swirl burner under piloted and premixed combustion conditions. Combust. Flame, (1995), 100, pp. 407-417

[5] T. Lieuwen, V. Yang, Combustion Instabilities in gas turbine engines: operational experience, fundamental mechanisms, and modelling, Progress in Astronautics and Aeronautics, AIAA publisher, 2005

[6] Y. Huang, S. Wang, V. Yang, Systematic analysis of lean-premixed swirl stabilised combustion, AIAA J. (2006), pp.724-740

[7] W. Pun, S.L. Palm, F.E.C. Culick, Combustion dynamics of an acoustically forced flame, Combust. Sci. Tech. (2003) 175, pp.499-521

[8] T. Poinsot, A. Trouve, D. Veynante, S. Candel, E. Esposito, Vortex driven acoustically coupled combustion instabilities, J. Fluid Mech. (1987) 177, pp.265-292 [9] K.C. Schadow, E. Gurmark, T.P. Parr, D.M. Parr, K.J. Wilson, J.E. Crump, Large scale coherent structures as drivers of combustion instability, Combut. Sci., Tech. (1989) 64, pp.167-186 
[10] T. Lieuwen, B.T. Zinn, The role of equivalence ratio oscillations in driving combustion instabilities in low Nox gas turbines, Proc. Combust. Inst. (1998) 27, pp.1809-1816

[11] V. Yang, W.E. Anderson, Liquid rocket engine combustion instability, Prog. Astro. Aero.169, AIAA publisher, 1992

[12] G.A. Richards, M.C. Janus, Characterisation of oscillations during premixed gas turbine combustors, J. Eng. Gas Turbine and Power (1997) 119, pp.776-782

[13] J.G. Lee, D.A. Santavicca, Experimental diagnostic for the study of combustion instabilities in lean-premixed combustors, J. Propu. and Power (2003) 19 (5), pp.735749

[14] K.H. Yu, A. Trouve, J.W. Daily, Low frequency pressure oscillations in model ramjet combustor, J. Fluid Mech. (1991) 232 (11), pp.47-72

[15] C. Presser, A.K. Gupta, H.G. Semerjain, Aerodynamics characteristics of swirling spray flame: Pressure jet atomiser, Combustion and Flame (1993) 92, pp.2544

[16] J.G. Lee, D.A. Santavicca, Effect of injection location on the effectiveness of an active control system using secondary fuel injection, Proc. Combust. Inst. (2000) 28, pp.739-746

[17] G.A. Richards, D.L. Straub, E.H. Robey, Passive control of combustion dynamics in stationary gas turbines, J. Propul. Power (2003) 19 (5), pp.795-809

[18] J.C. Oefelein, Large eddy simulation of turbulent combustion processes in propulsion and power systems, Prog Aero Sciences (2006) 42, pp. 2-37

[19] N. Patel, S. Menon, Simulation of spray-turbulence-chemistry interactions in a lean direct injection combustor, Combust. Flame (2008) 153, pp. 228-257 
[20] A. Roux, L.Y.M. Gicquel, Y. Sommerer, T.J. Poinsot, Large eddy simulation of mean and oscillating flow in a side dump ramjet combustor, Combust Flame (2008) 152, pp. $154-176$

[21] H, Pitsch, Large eddy simulation of turbulent combustion, Ann. Rev. Fluid Mech. (2006) 38, pp. 453-482

[22] A.W. Cook, J.J. Riley, A subgrid model for equilibrium chemistry in turbulent flows, Phy. Fluids (1994) 6(8), pp. 2868-2870

[23] N. Branley, W.P. Jones, Large eddy simulation of turbulent non-premixed flames, Combust. Flame (2001) 127, pp. 1917-1934

[24] R. Venkatramanan, H. Pitsch, Large eddy simulation of bluff body stabilized non-premixed flame using a recursive filter refinement procedure, Combust. Flame (2005) 142, pp. 329-347

[25] A. Kempf, R.P. Lindstedt, J. Janika, Large eddy simulation of bluff body stabilized non-premixed flame, Combust. Flame (2006)144, pp. 170-189

[26] C.D. Pierce, P. Moin, Progress variable approach for LES of non-premixed turbulent combustion, J Fluid Mech. (2004) 504, pp. 73-97

[27] S. Navarro-Marteniz, A. Kronenburg, Investigation of LES-CMC modelling in a bluff-body stabilised non-premixed flame, Proc. EU Combust. Meeting, 1-6, 2005 [28] P.A. Mcmurtry, S. Menon, A. Kerstein, A linear eddy subgrid model for turbulent reacting flows: application to hydrogen air combustion, Proc Combust Inst, (1992) 25, pp. 271-278

[29] Y. Huang, H.G. Sung, S.Y. Hsieh, V. Yang, Large eddy simulation of combustion dynamics of lean-premixed swirl stabilised combustor, J. Prop. And Power (2003) 19, pp.782-794 
[30] W.W. Kim, S. Syed, Large eddy simulation needs for gas turbine combustor design, AIAA paper (2004), 2004-0331

[31] F. Di Mare, W. Jones, K. Menzies, Large eddy simulation of a model gas turbine combustor, Combust. Flame (2004) 137(3), pp.278-294

[32] L. Selle G. Lartigue T. Poinsot, R. Koch, K. Schildmacher, W. Krebs et al. Compressible large eddy simulation of turbulent combustion in complex geometry on unstructured meshes, Combust. Flame (2004) 137, pp.489-505

[33] F.F.Grinstein, C. Fureby, LES studies of the flow in a swirl gas combustor, Proc. Combust. Inst. (2005) 30, pp. 1791-1798

[34] K. Mahesh, G. Constantinescu, S. Apte, G. Iaccarineo, F. Ham, P. Moin, Large eddy simulation of turbulent flow in complex geometries, ASME J. App. Mech. (2006) 73, pp.374-381.

[35] C. Fureby, F.F. Grinstein, G. Li, E.J. Gutmark, An experimental and computational study of a multi-swirl gas turbine combustor, Proc. Combust. Inst (2007) 31 (2), pp.3107-3114

[36] M. Bioleau, G. Staffelbach, B. Cuenot, T. Poinsot, C. Berat, LES of an ignition sequence in gas turbine engine, Combust. Flame (2008) 154, pp.2-22

[37] G.L. Boudier, Y.M. Gicquel, T.J. Poinsot, Effects of mesh resolution on large eddy simulations of reacting flows in complex geometry combustors, Combust. Flame (2008) 155, pp.196-214

[38] P.A.M.Kalt, Y.M, Al-Abdeli, A.R. Masri, R.S.Barlow, Swirling turbulent nonpremixed flames of methane: Flow field and compositional structure, Proc. Combust. Inst. (2002) 29, pp.1913-1919

[39] Y.M. Al-Abdeli, A.R.Masri, Stability characteristics and flowfields of turbulent non-premixed swirling flames, Combust. Theory. Model. (2003) 7, pp.731-766 
[40] A.R.Masri, P.A.M.Kalt, R.S.Barlow, The compositional structure of swirl stabilised turbulent non-premixed flames, Combust. Flame (2004) 137, pp.1-37

[41] Y.M, Al-Abdeli, A.R. Masri, G.R. Marquez, G.R, S. Staner, Time-varying behaviour of turbulent swirling nonpremixed flames, Combust. Flame (2006) 146, pp. 200-214

[42] H. El-Asrag, S. Menon, Large eddy simulation of bluff-body stabilised swirling non-premixed flame, Proc. Combust. Inst. (2007) 31, pp. 1747-1754

[43] S. James, J. Zhu, M.S. Anand, Large eddy simulation of turbulent flames using filtered density function method, Proc. Combust. Inst. (2007) 31, pp. 1737-1745

[44] W. Malalasekera K.K.J. Ranga Dinesh, S.S. Ibrahim, M.P. Kirkpatrick, Large eddy simulation of isothermal turbulent swirling jets, Combust. Sci. Tech. (2007) 179, pp. $1481-1525$

[45] W. Malalsekera, K.K.J. Ranga Dinesh, S.S. Ibrahim, A.R. Masri, LES of recirculation and vortex breakdown in swirling flames, Combust. Sci. Tech. (2008) 180, pp. 809-832

[46] A. Kempf, W. Malalasekera, K.K.J. Ranga Dinesh, O. Stein, Large eddy simulation with swirling non-premixed flames with flamelet model: A comparison of numerical methods. Flow Turb. Combust. (2008) 81, pp.523-561

[47] K.K.J.Ranga Dinesh, M.P.Kirkpatrick, Study of jet precession, recirculation and vortex breakdown in turbulent swirling jets using LES, Int. J. Comput. Fluids (2009), 38, pp.1232-1242

[48] J. Smagorinsky, General circulation experiments with the primitive equations. M. Weather Review. (1963) 91, pp. 99-164

[49] U. Piomelli, J. Liu, Large eddy simulation of channel flows using a localized dynamic model. Phy. Fluids (1995) 7, pp. 839-848 
[50] H. Pitsch, A C++ computer program for 0-D and 1-D laminar flame calculations, RWTH, Aachen, 1998

[51] C.T. Bowman, R.K. Hanson, D.F. Davidson, W.C. Gardiner, V. Lissianki, G.P.Smith, D.M. Golden, M. Frenklach, M. Goldenberg, GRI 2.11, 2006

[52] M.P. Kirkpatrick, S.W. Armfield, J.H. Kent, A representation of curved boundaries for the solution of the Navier-Stokes equations on a staggered threedimensional Cartesian grid, J. of Comput. Phy. (2003) 184, pp. 1-36.

[53] M.P. Kirkpatrick, S.W. Armfield, A.R. Masri, S.S. Ibrahim, Large eddy simulation of a propagating turbulent premixed flame, Flow, Turb. Combust.(2003) 70 (1), pp.1-19.

[54] M.P. Kirkpatrick, S.W. Armfield, Experimental and large eddy simulation results for the purging of a salt water filled cavity by an overflow of fresh water, Int. J. of Heat and Mass Trans. (2005) 48 (2), pp.341-359

[55] K.K.J. Ranga Dinesh, Large eddy simulation of turbulent swirling flames. PhD Thesis, Loughborough University, UK. 2007

[56] B.P. Leonard, SHARP simulation of discontinuities in highly convective steady flows, NASA Tech. Memo. (1987), Vol. 100240, 1987

[57] Y.M. Al-Abdeli, A.R. Masri, Precession and recirculation in turbulent swirling isothermal jets, Combust. Sci. Tech. (2004) 176, pp. 645-665 


\section{Figure Captions}

Figure 1: Schematic drawing of the Sydney swirl burner

Figure 2: Snapshots of the filtered temperature for flame SMH1

Figure 3: Snapshots of the filtered temperature for flame SMH2

Figure 4: Snapshots of the filtered temperature for flame SMH3

Figure 5: Comparison of mean axial velocity for flame SMH1 (left side) and SMH2

(right side). Line denotes LES data and symbols denote experimental data.

Figure 6: Comparison of mean swirling velocity for flame SMH1 (left side) and SMH2 (right side). Line denotes LES data and symbols denote experimental data.

Figure 7: Comparison of rms axial velocity for flame SMH1 (left side) and SMH2 (right side). Line denotes LES data and symbols denote experimental data.

Figure 8: Comparison of mean temperature for flame SMH1 (left side) and SMH2 (right side). Line denotes LES data and symbols denote experimental data.

Figure 9: Comparison of $\mathrm{CO}_{2}$ for flame SMH1 (left side) and SMH2 (right side). Line denotes LES data and symbols denote experimental data. 
Figure 10: Comparison of $\mathrm{CO}$ for flame SMH1 (left side) and SMH2 (right side). Line denotes LES data and symbols denote experimental data.

Figure 11: Mode I instability in flame SMH1 identified using LES

Figure12. Mode I instability of flame SMH1 in a plane perpendicular to the centreline

Figure 13: Power spectrum of the flame SMH1 at spatial jet locator

Figure 14: Mode I instability in flame SMH2 identified using LES

Figure15. Mode I instability of flame SMH2 in a plane perpendicular to the centreline

Figure 16: Power spectrum of the flame SMH2 at spatial jet locator

Figure 17: Mode II instability in flame SMH3 identified using LES

Figure 18: Power spectrum of the flame SMH3 at envelope of the recirculation zone 
Figures

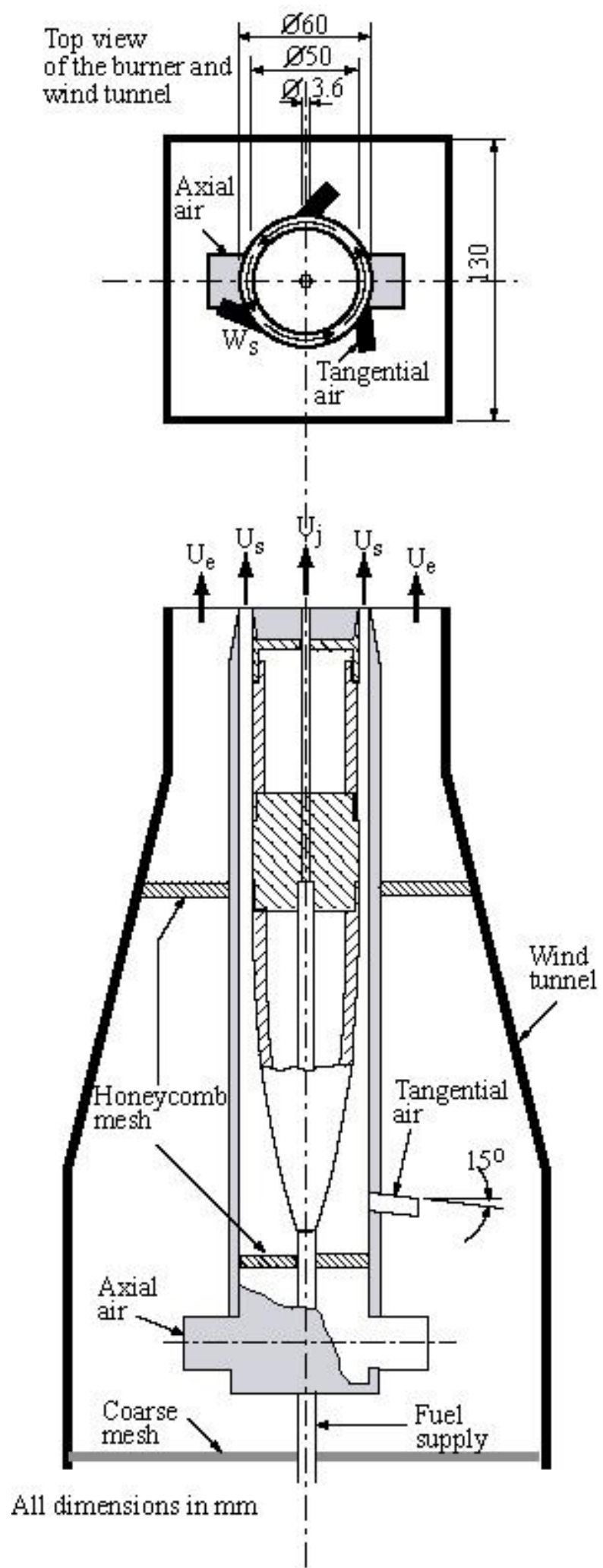

Figure 1. Schematic drawing of the Sydney swirl burner 


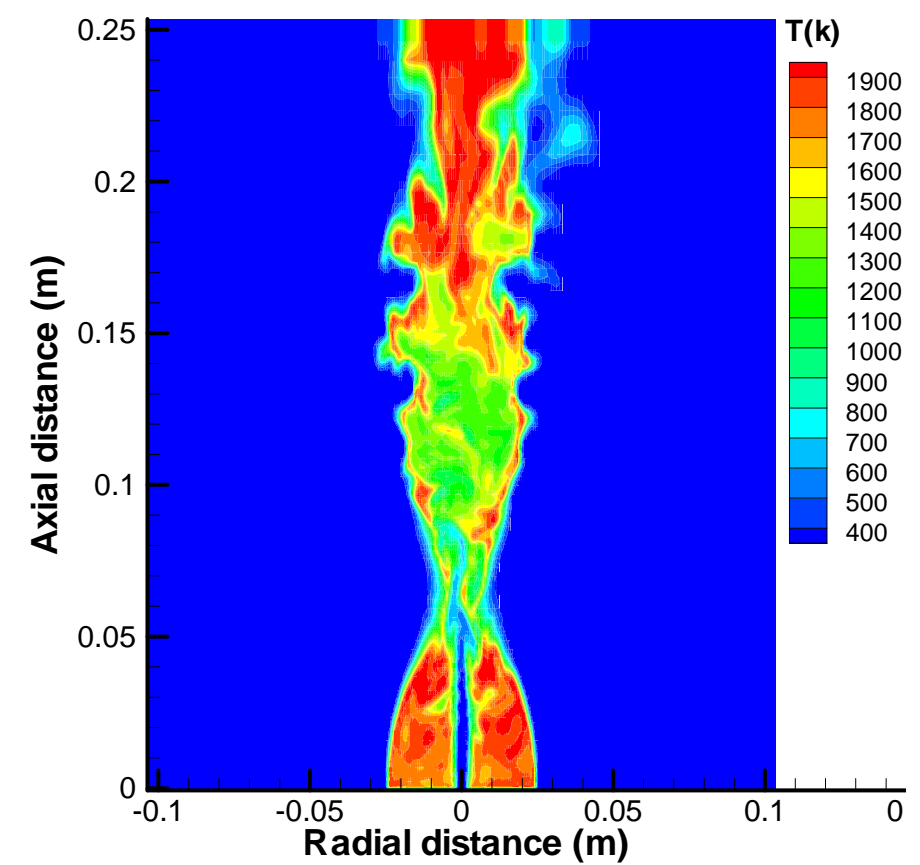

Figure 2: Snapshots of the filtered temperature for flame SMH1

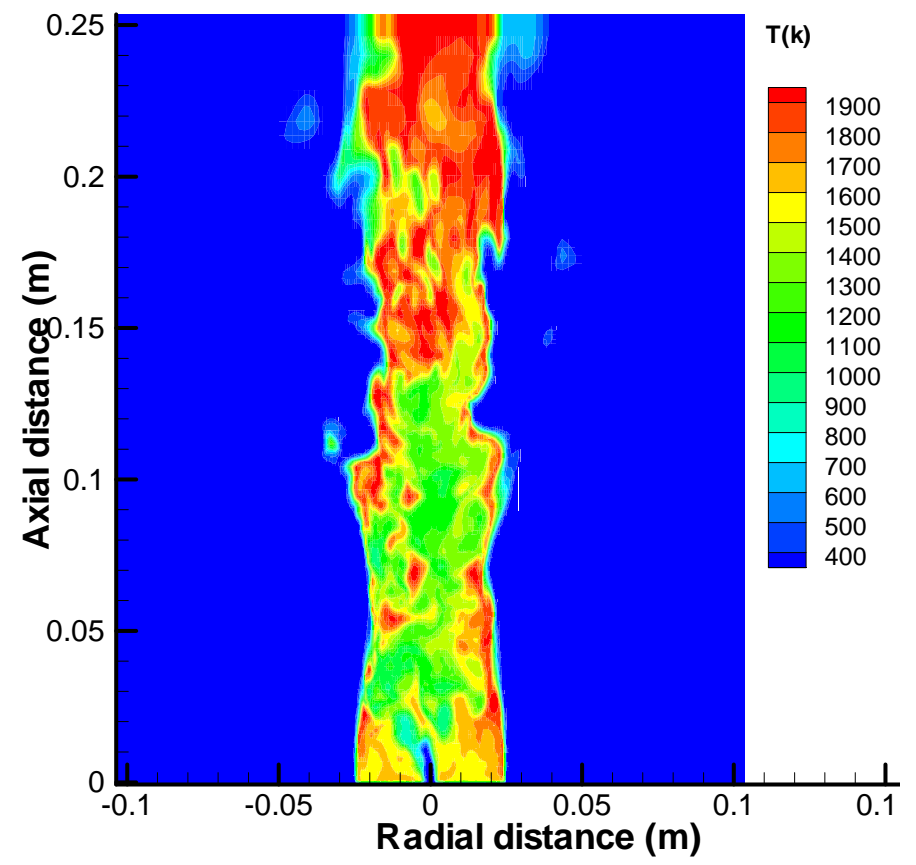

Figure 3: Snapshots of the filtered temperature for flame SMH2 


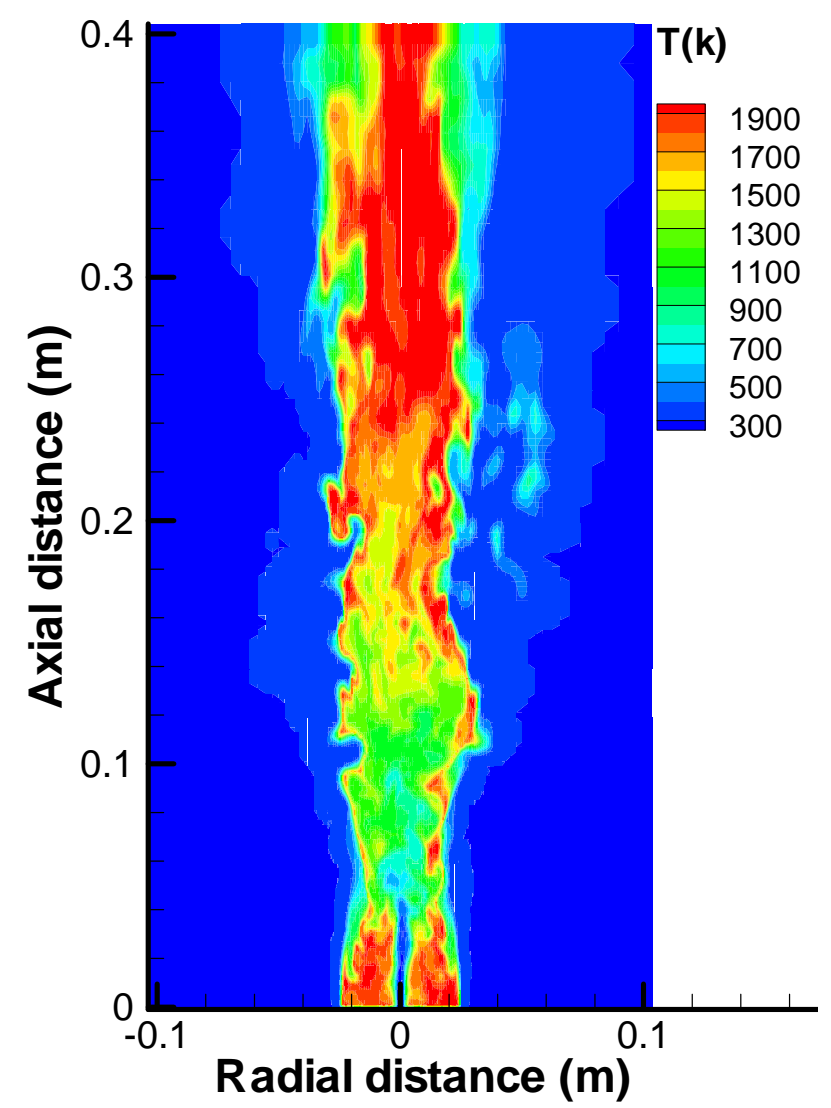

Figure 4: Snapshots of the filtered temperature for flame SMH3 

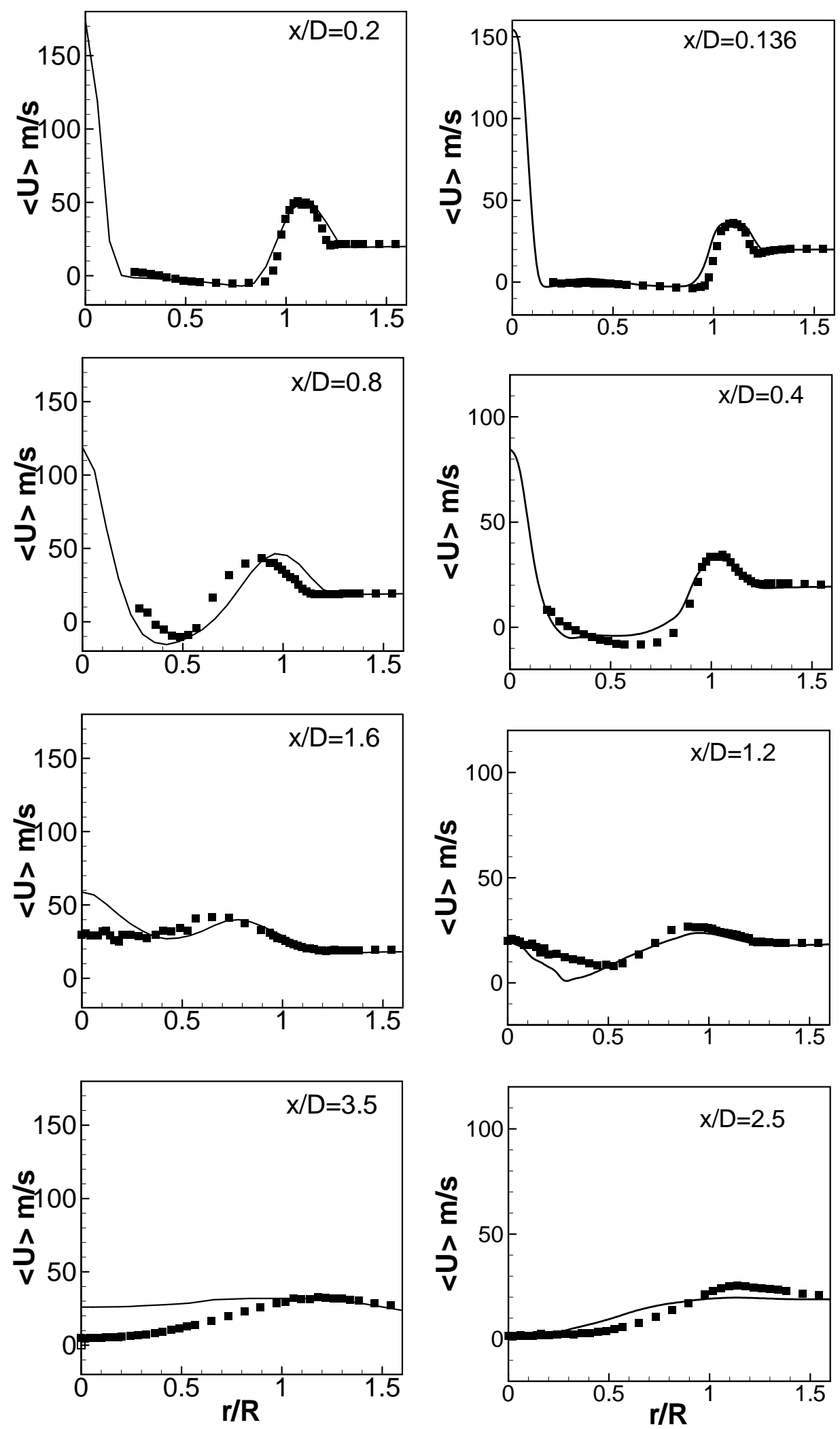

Figure 5: Comparison of mean axial velocity for flame SMH1 (left side) and SMH2 (right side). Line denotes LES data and symbols denote experimental data. 

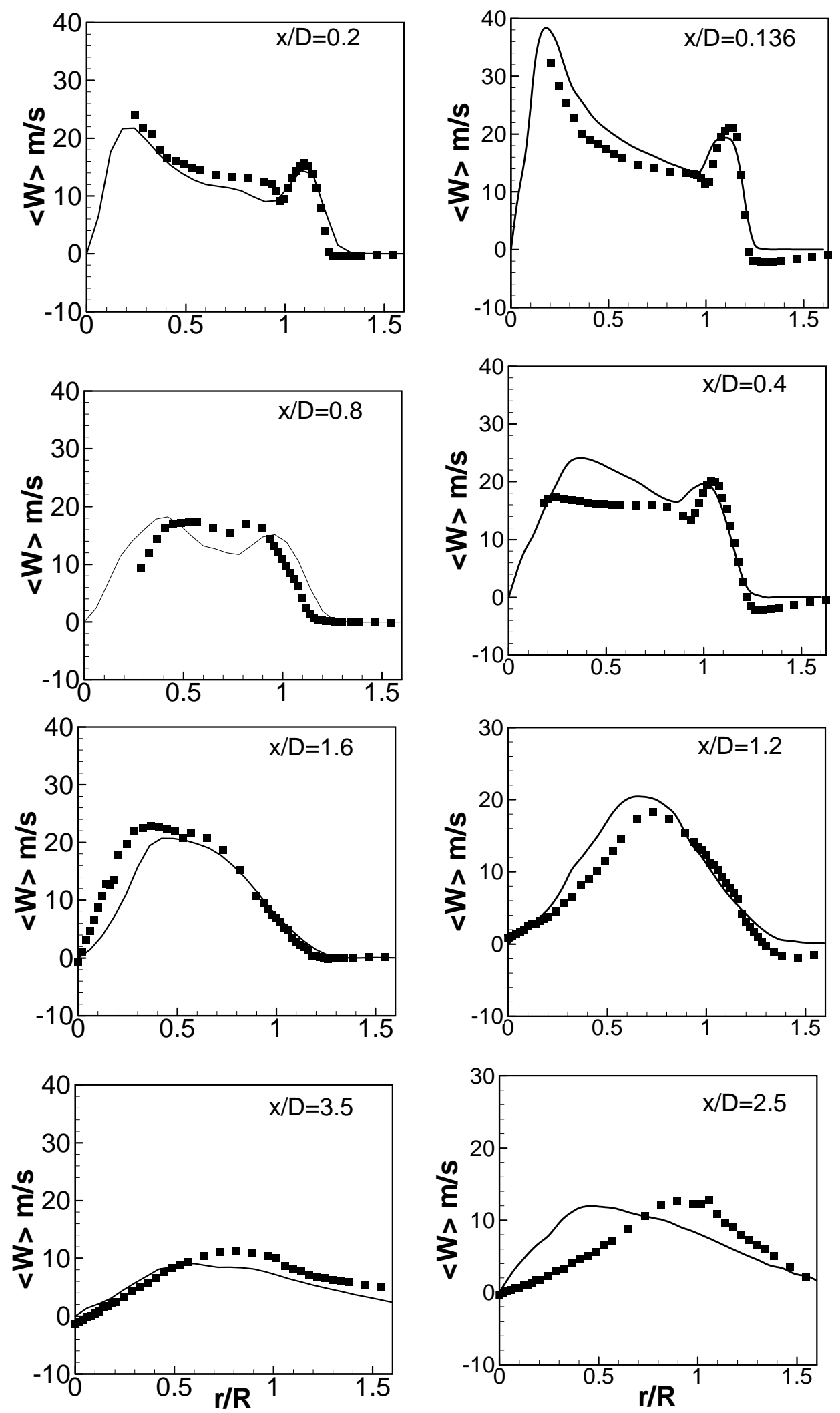

Figure 6: Comparison of mean swirling velocity for flame SMH1 (left side) and SMH2 (right side). Line denotes LES data and symbols denote experimental data. 

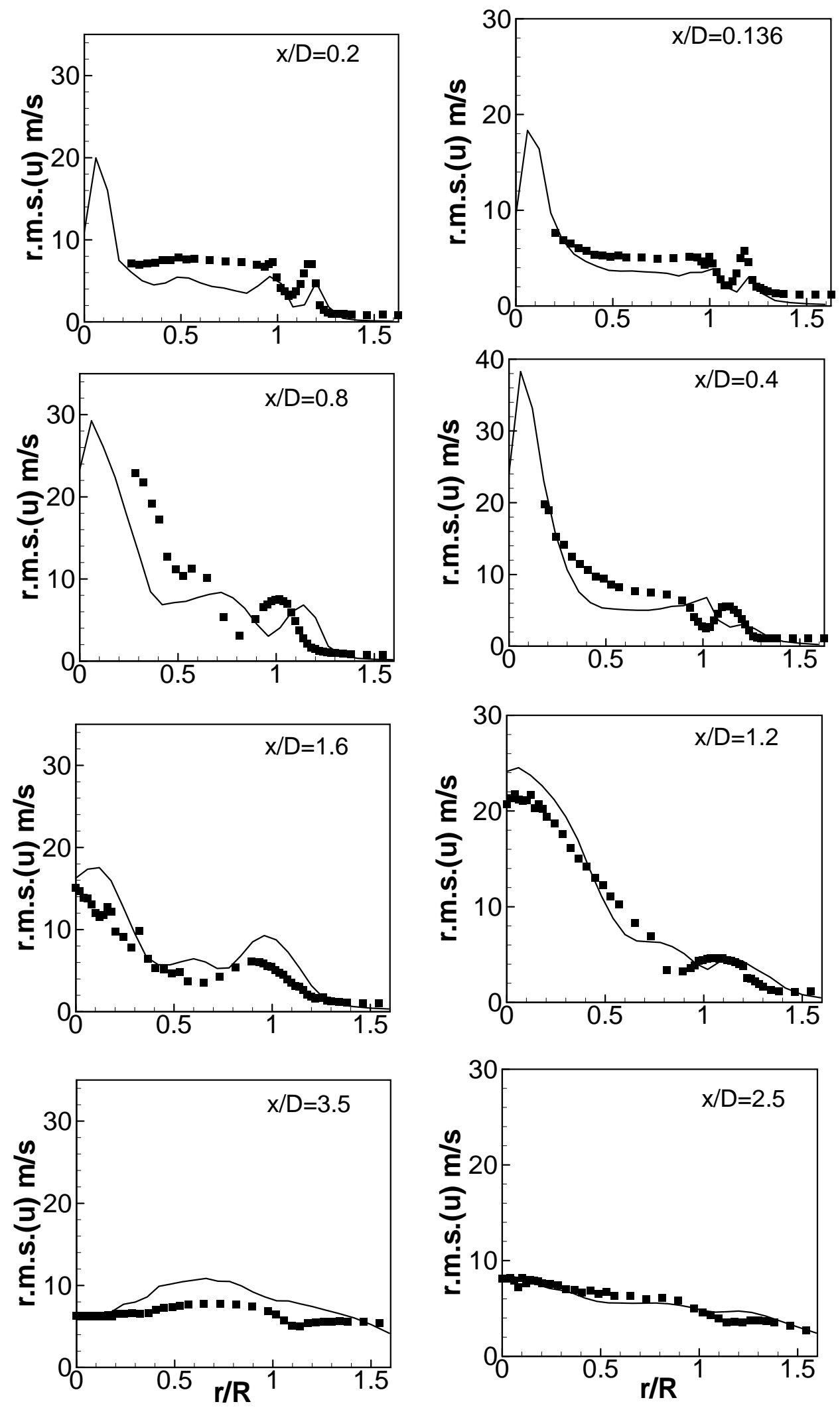

Figure 7: Comparison of rms axial velocity for flame SMH1 (left side) and SMH2 (right side). Line denotes LES data and symbols denote experimental data. 

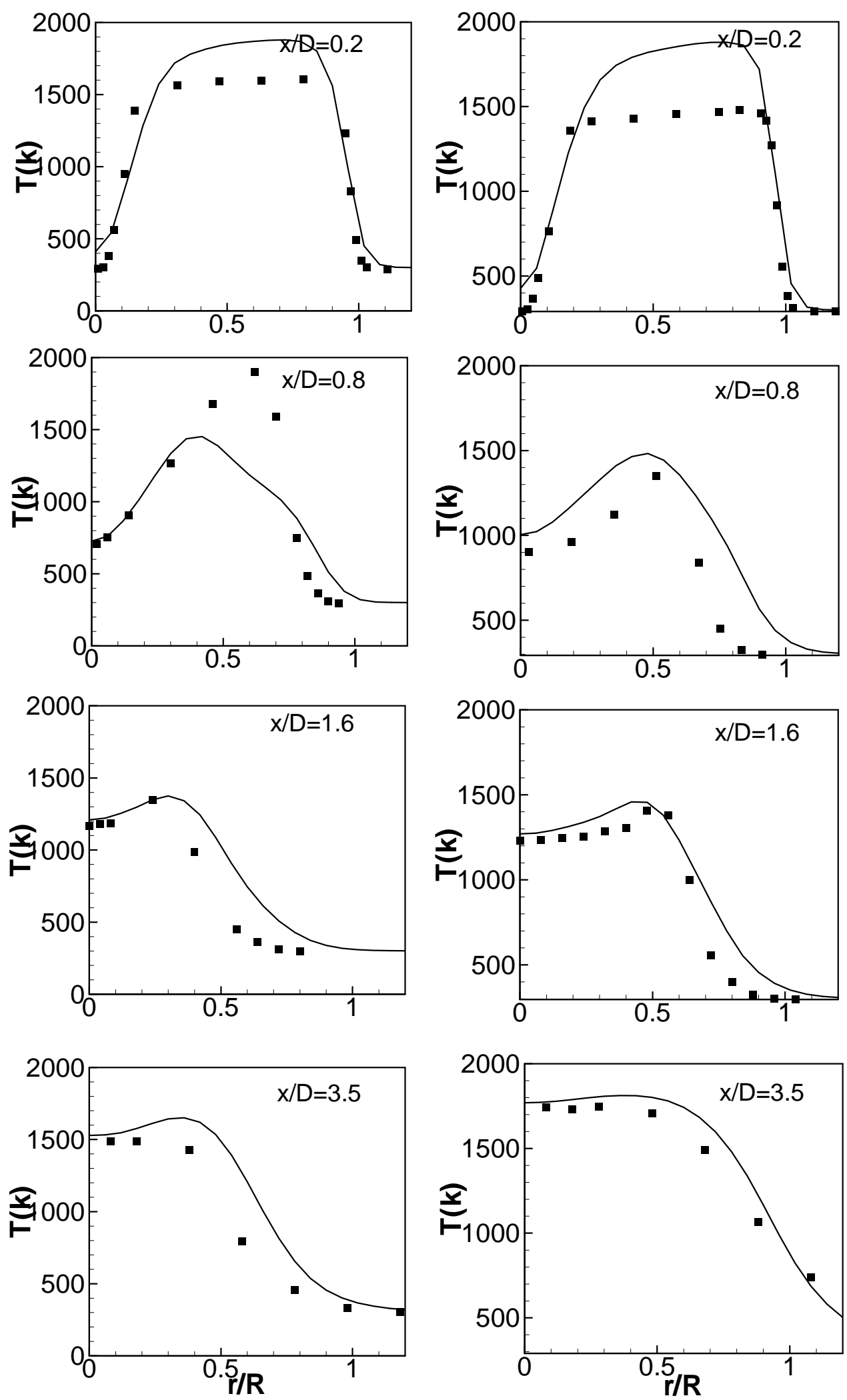

Figure 8: Comparison of mean temperature for flame SMH1 (left side) and SMH2

(right side). Line denotes LES data and symbols denote experimental data. 

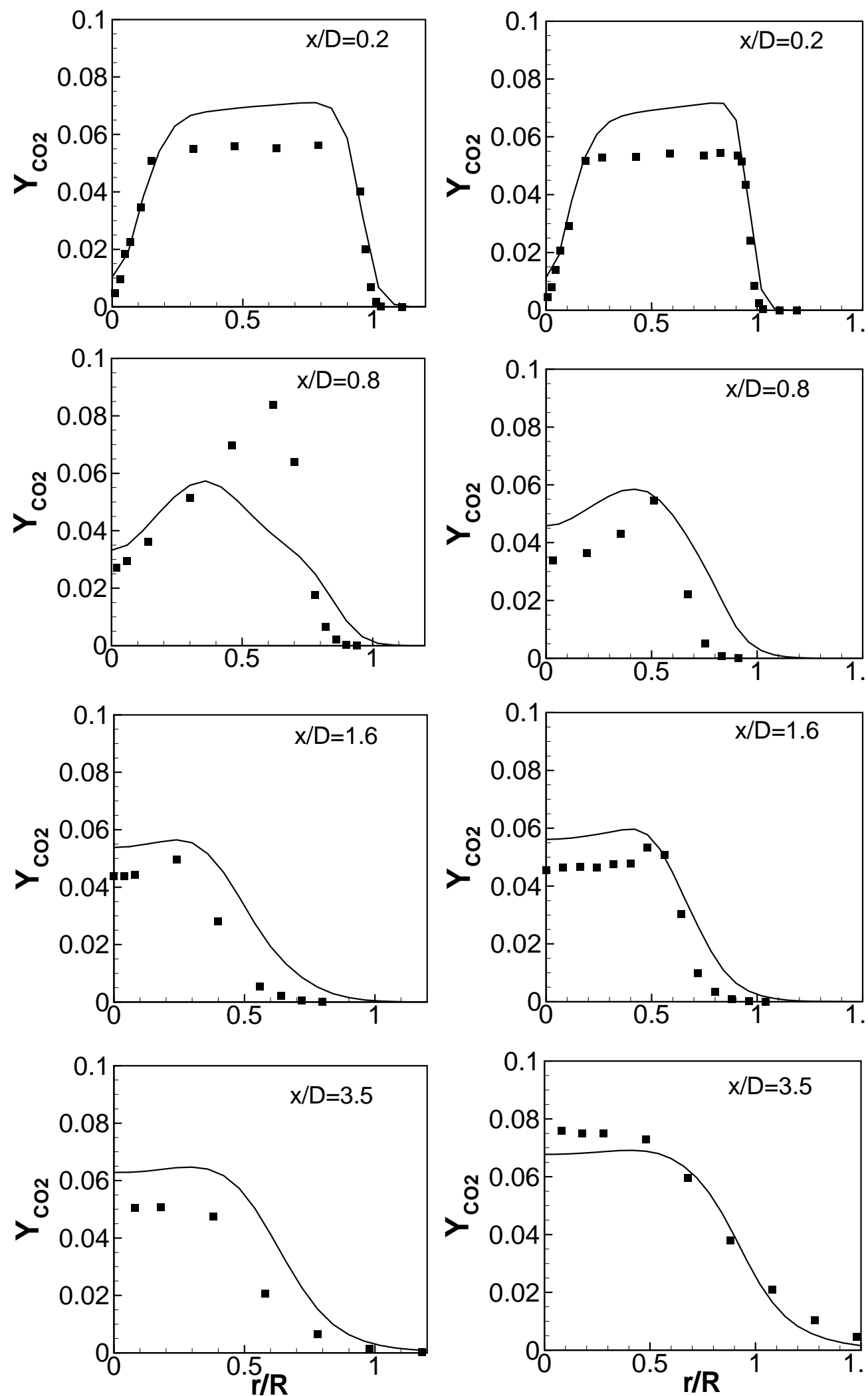

Figure 9: Comparison of $\mathrm{CO}_{2}$ for flame SMH1 (left side) and SMH2 (right side). Line denotes LES data and symbols denote experimental data 

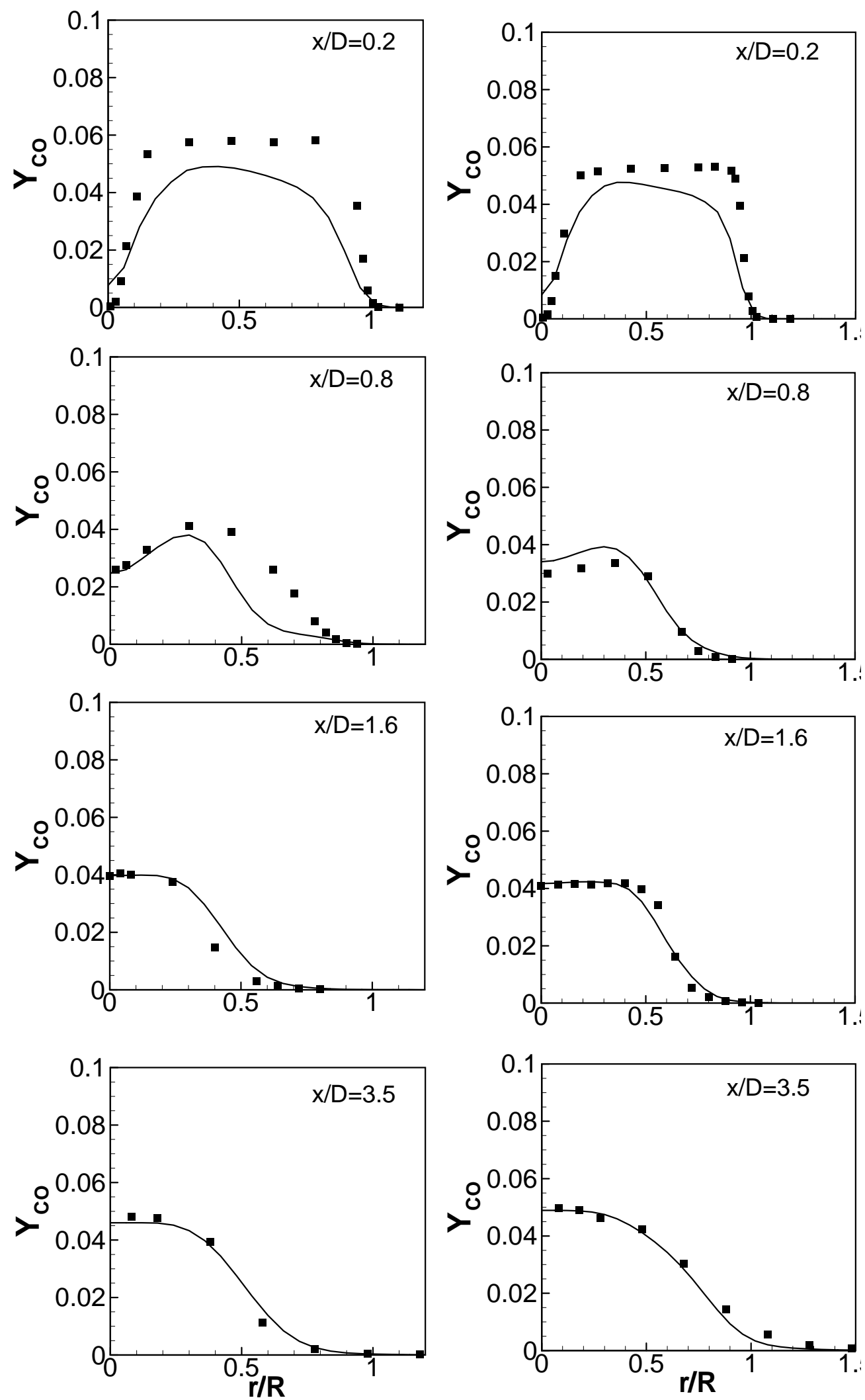

Figure 10: Comparison of CO for flame SMH1 (left side) and SMH2 (right side). Line denotes LES data and symbols denote experimental data 


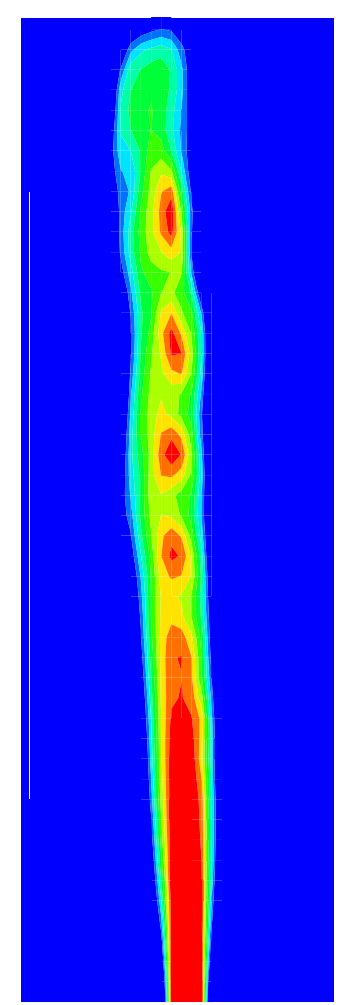

$\mathrm{a}$

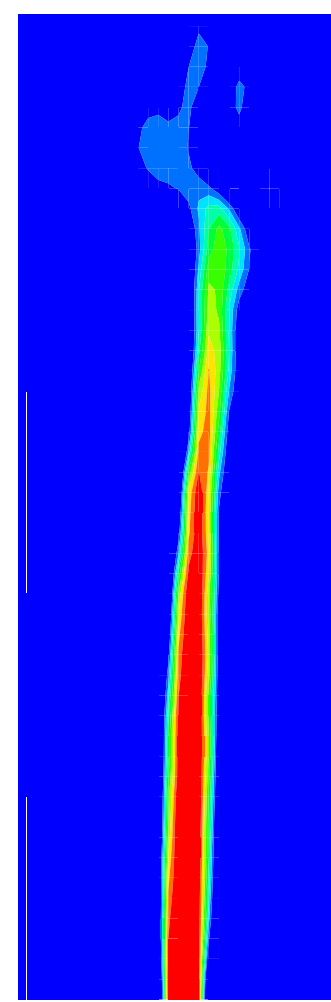

e

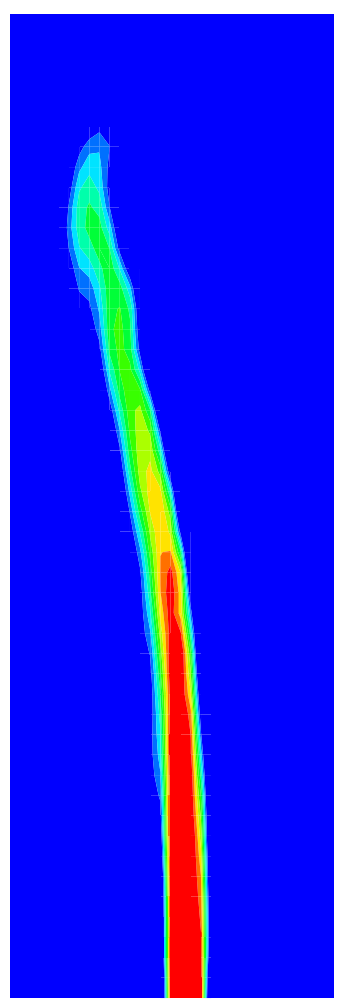

b

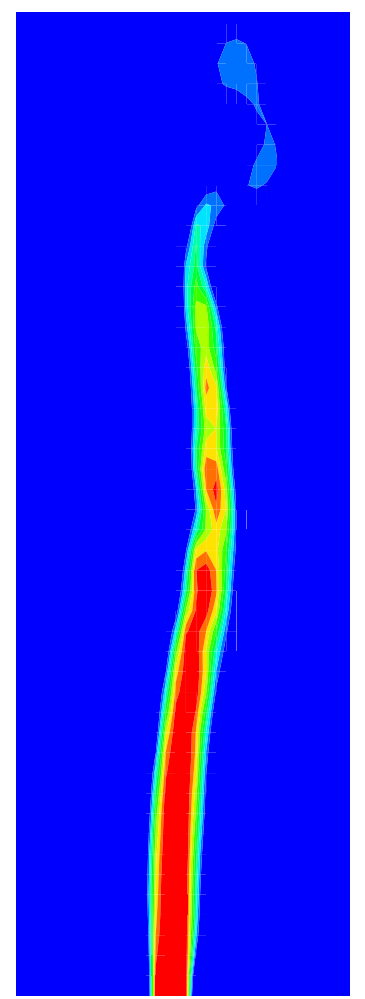

$\mathrm{f}$

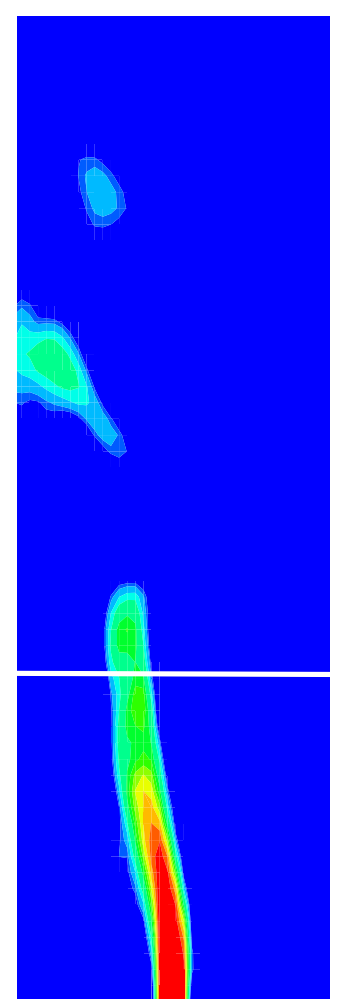

C

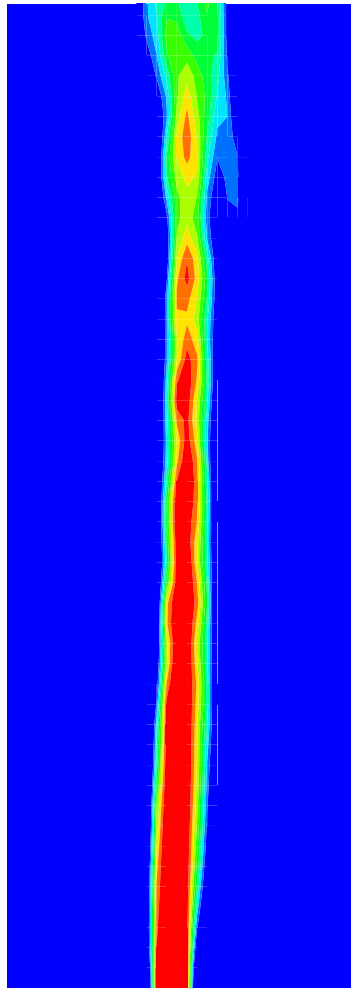

d

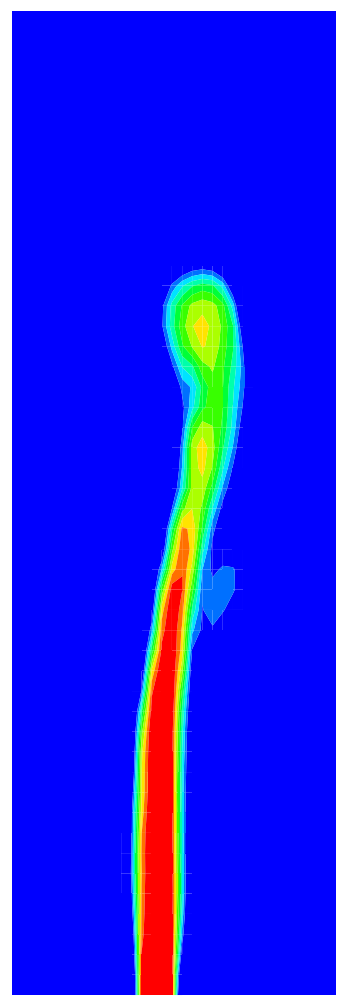

g

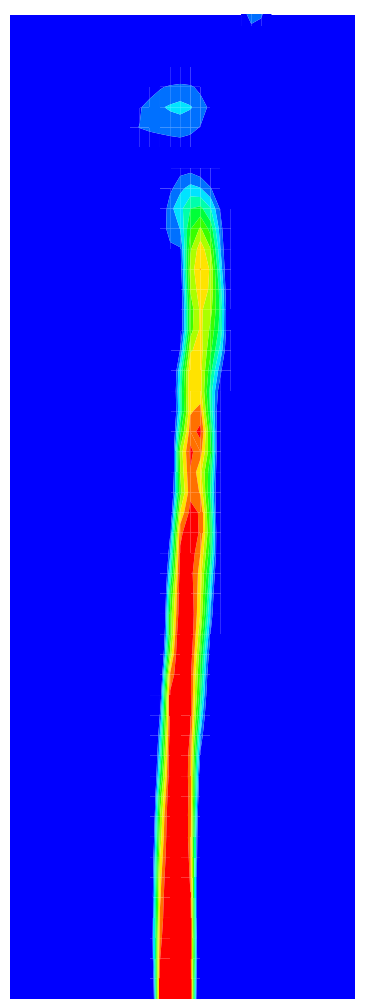

h

Figure 11. Mode 1 instability in flame SMH1 identified using LES visualised by filtered axial velocity 


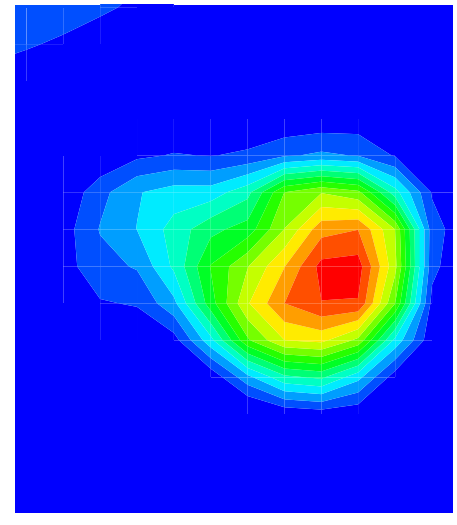

aa

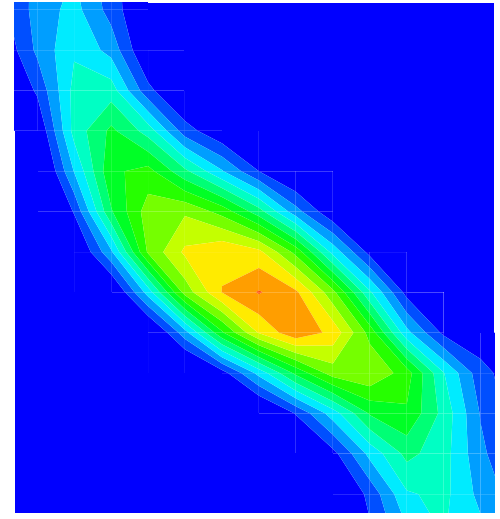

CC

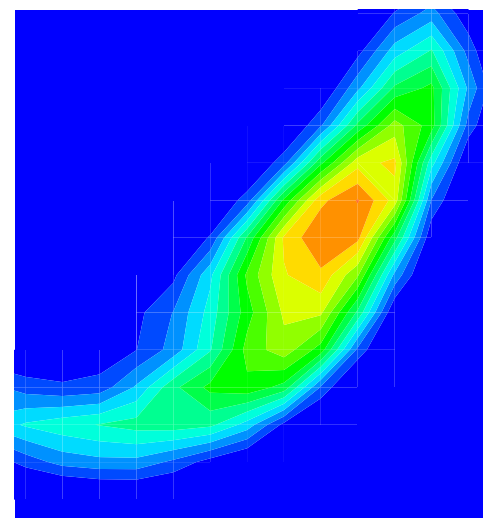

gg

Figure 12. Mode I instability of flame SMH1 in a plane perpendicular to the centreline

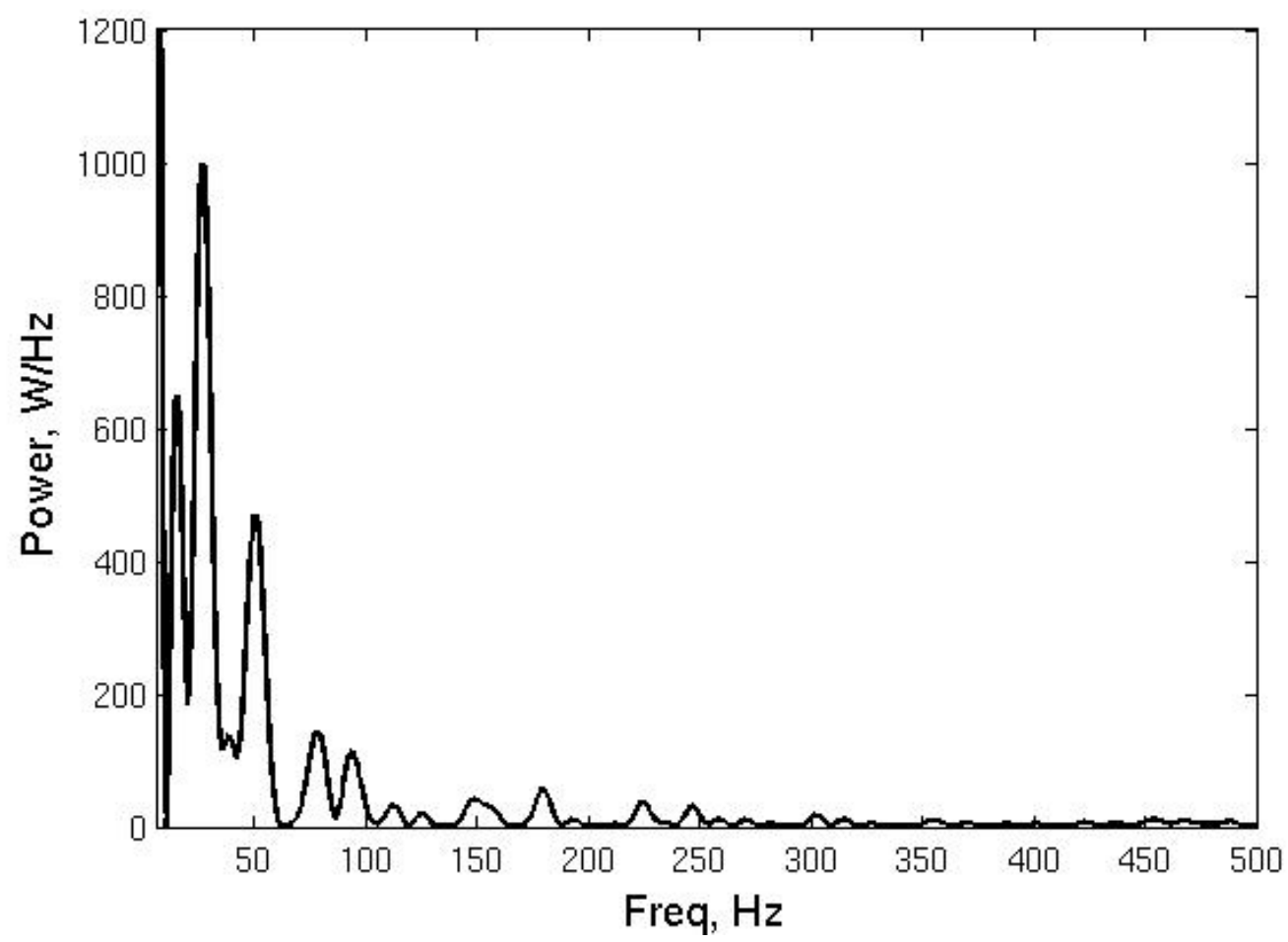

Figure 13. Power spectrum of the flame SMH1 at spatial jet locator 


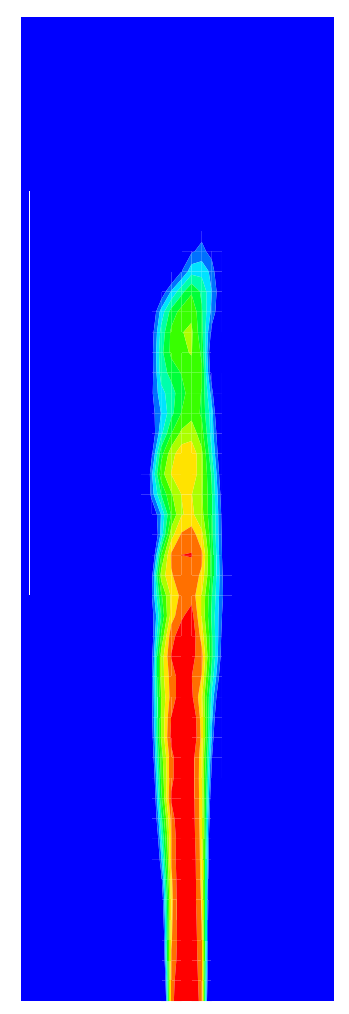

a

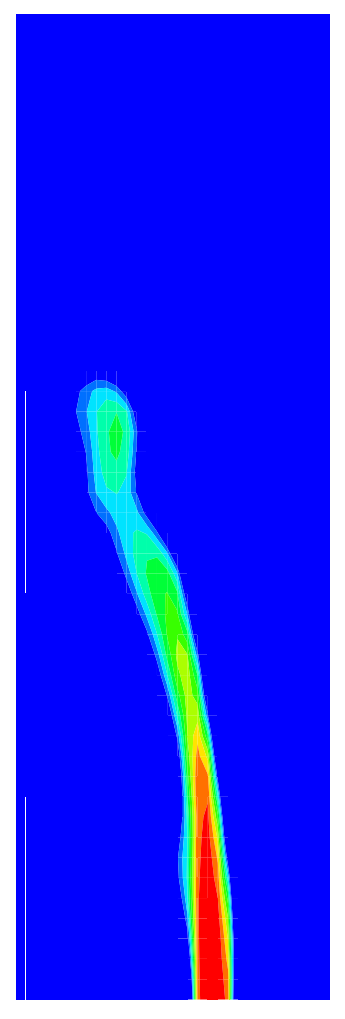

e

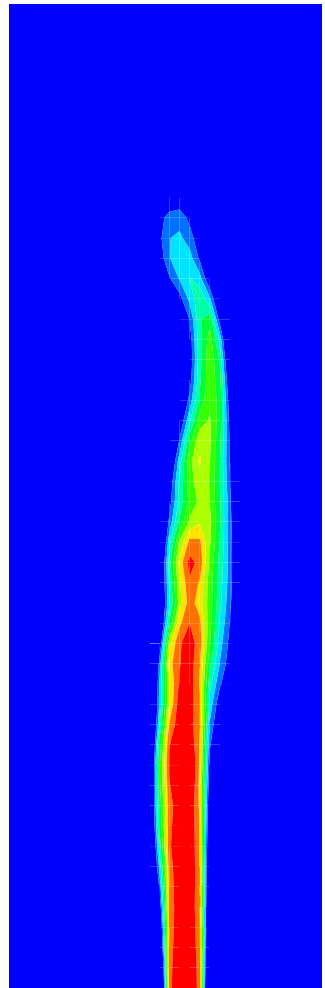

b

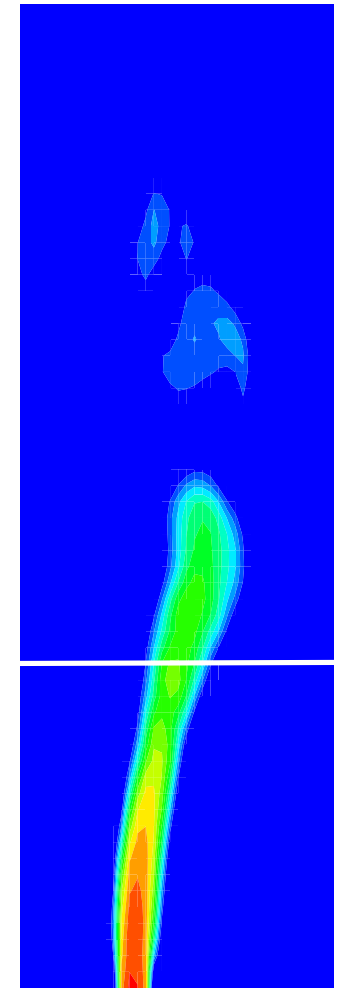

C

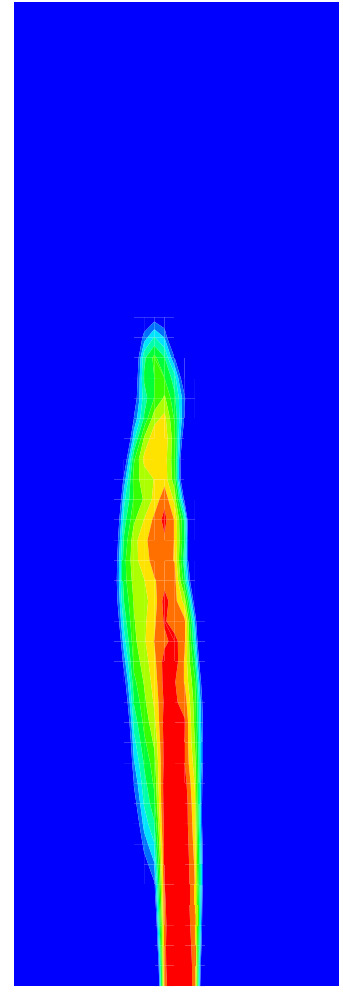

d

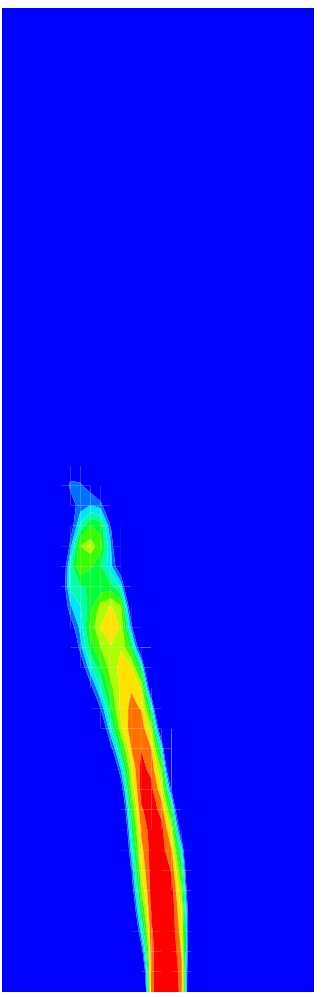

f

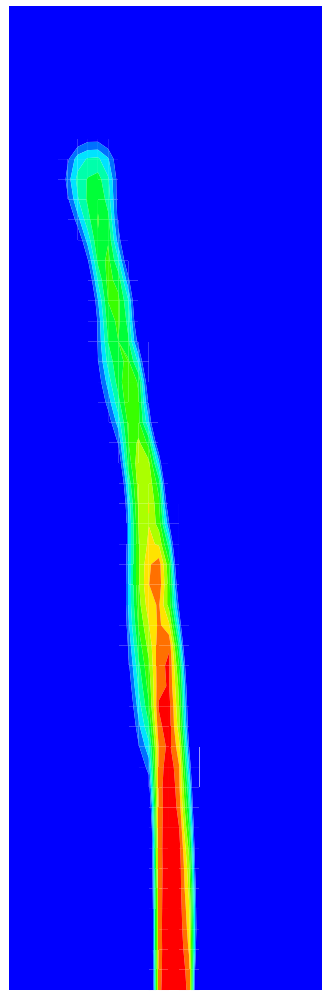

g

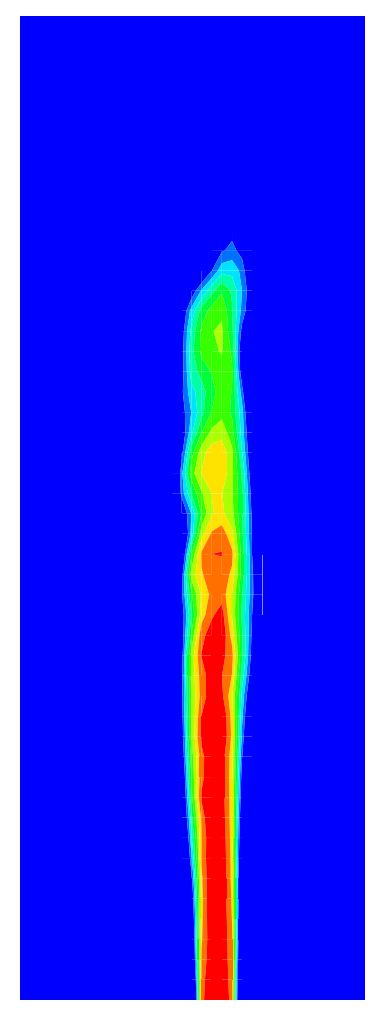

$\mathrm{h}$

Figure 14. Mode 1 instability in flame SMH2 identified using LES visualised by filtered axial velocity 


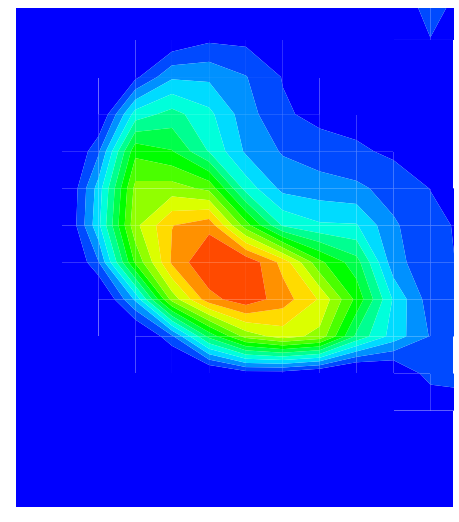

aа

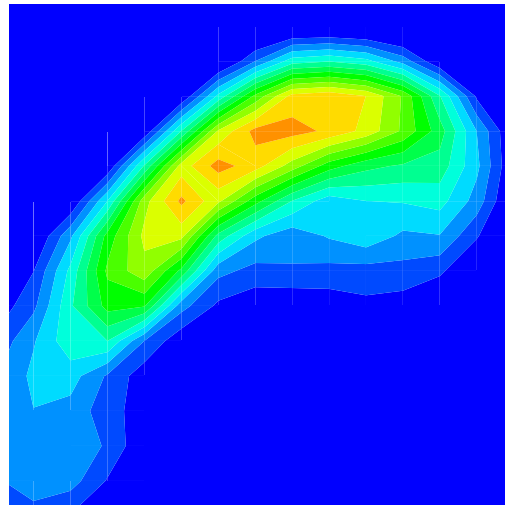

CC

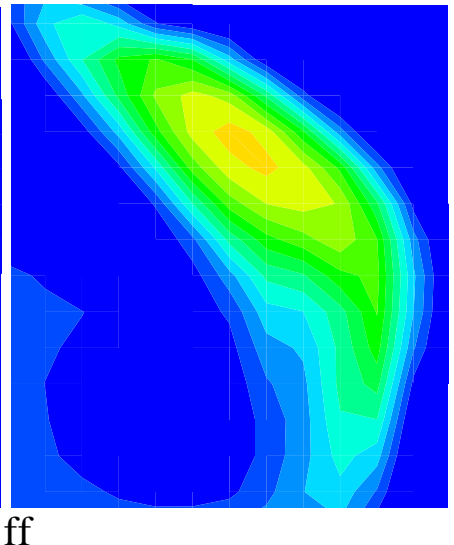

ff

Figure 15. Mode I instability of flame SMH2 in a plane perpendicular to the centreline

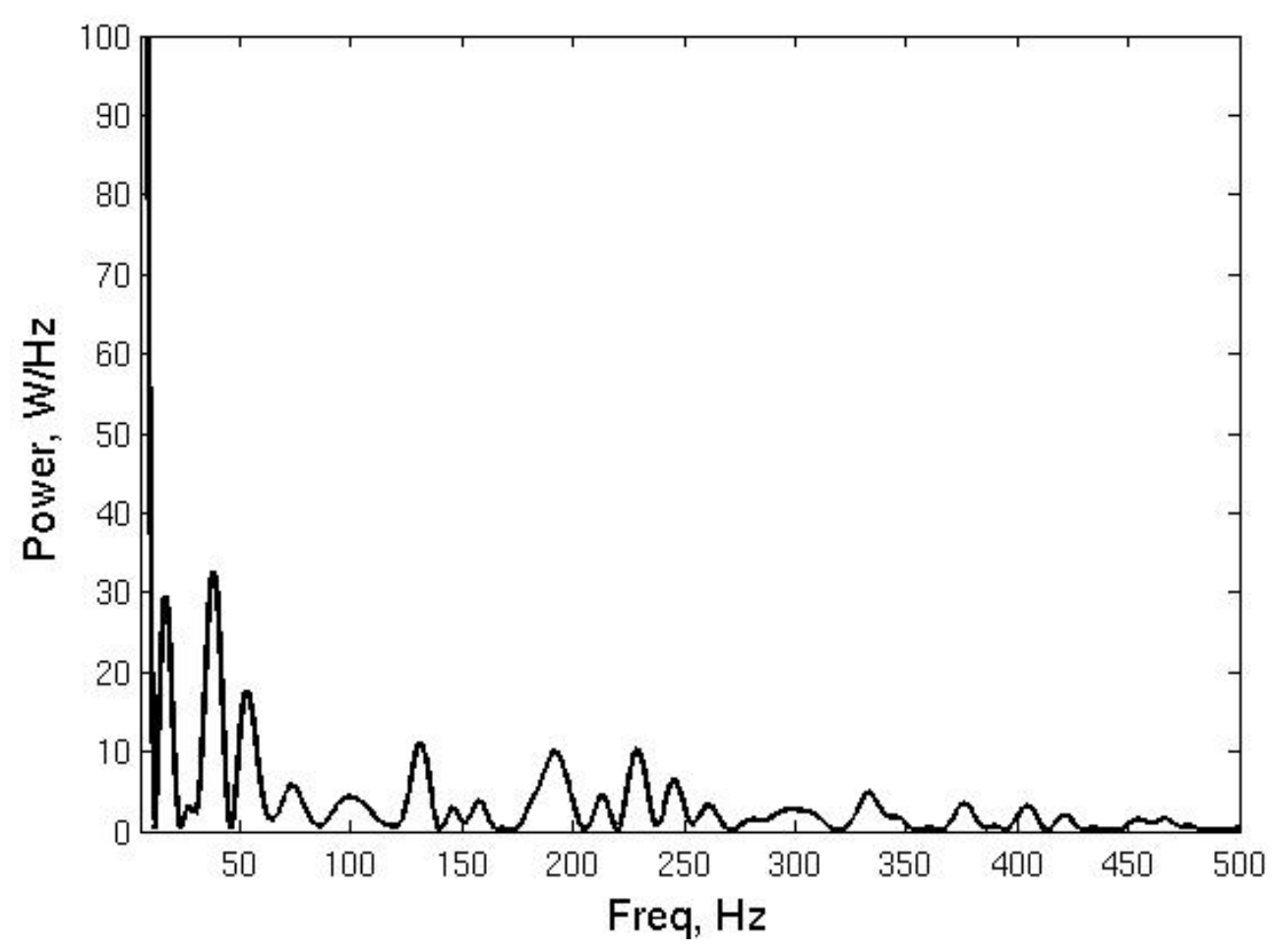

Figure 16. Power spectrum of the flame SMH2 at spatial jet locator 

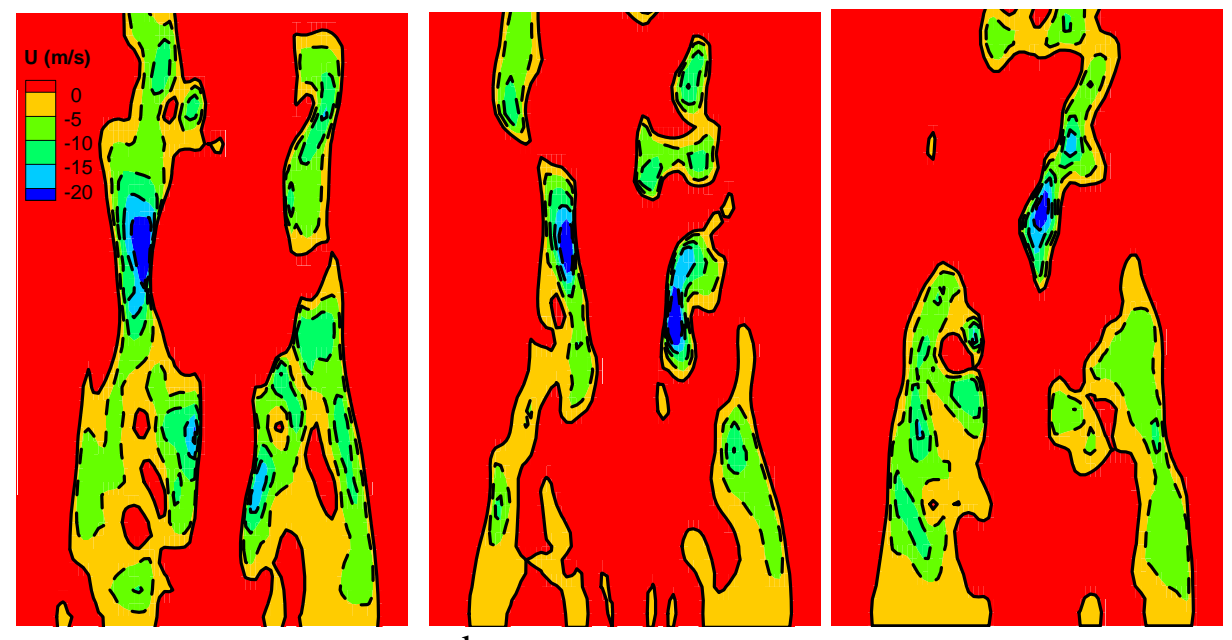

a

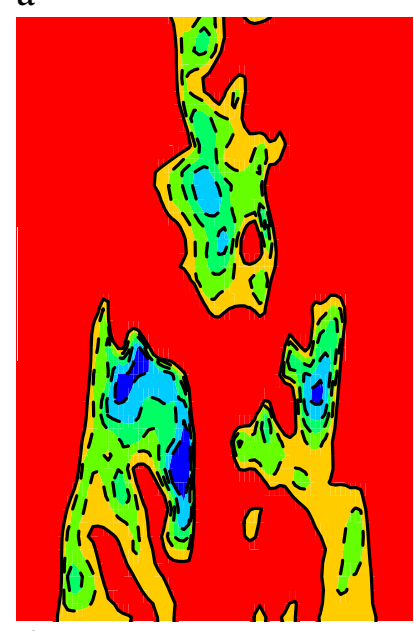

b

C
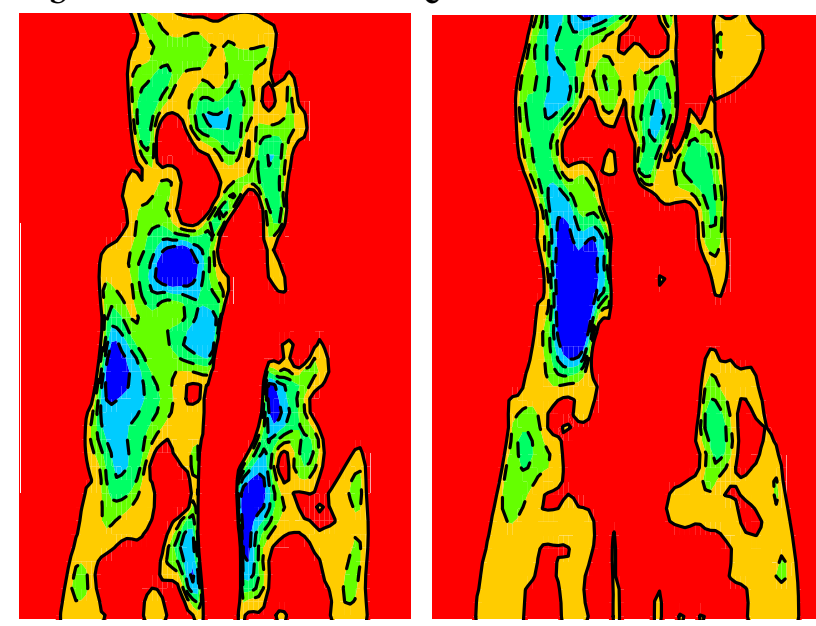

e

$\mathrm{f}$

Figure 17. Mode II instability in flame SMH3 identified using LES visualised by filtered axial velocity 


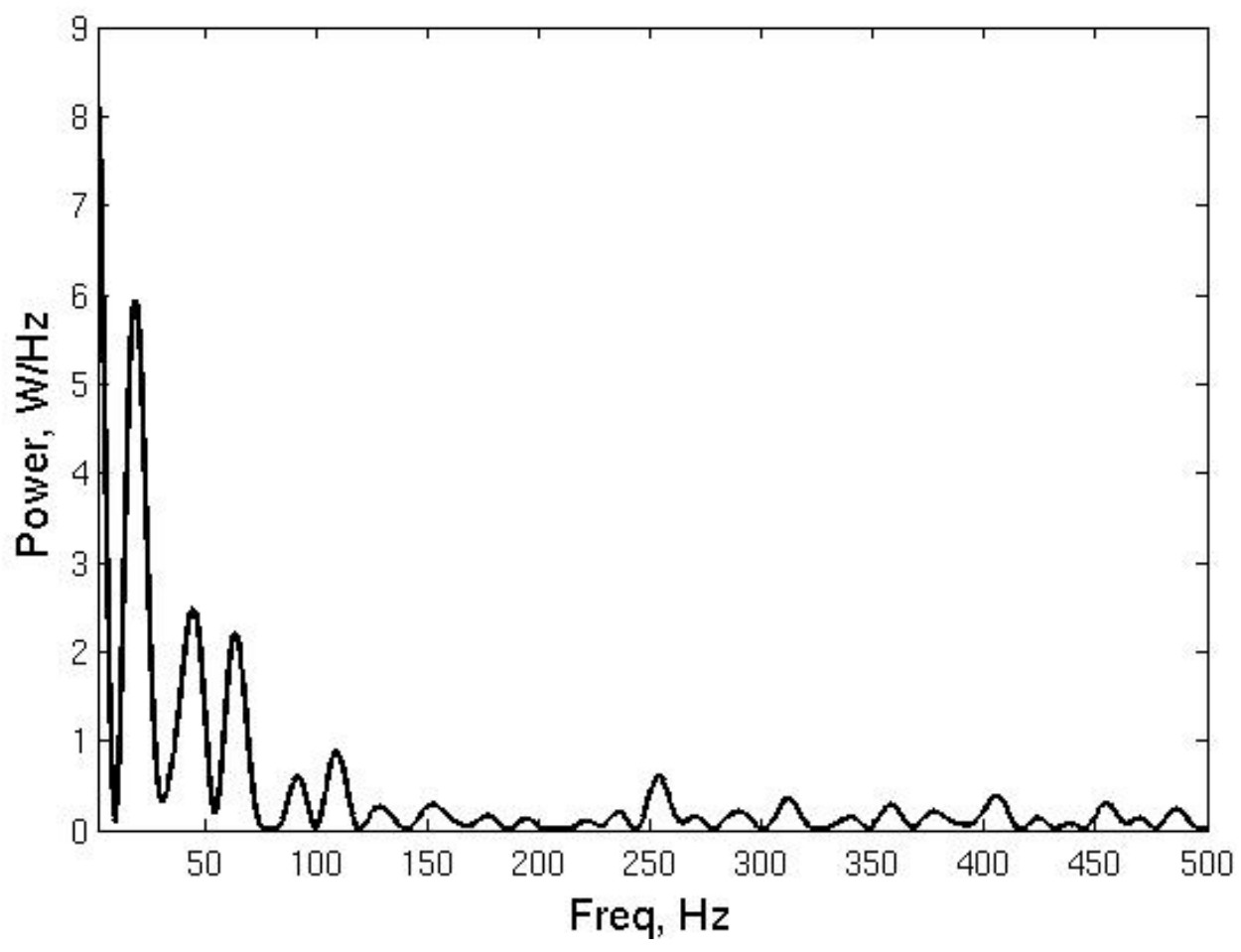

Figure 18. Power spectrum of the flame SMH3 at envelope of the recirculation zone 